\title{
Anti-neurofascin-155 IgG4 antibodies prevent paranodal complex formation in vivo
}

\author{
Constance Manso, ${ }^{1,2}$ Luis Querol, ${ }^{3,4}$ Cinta Lleixà, ${ }^{3,4}$ Mallory Poncelet, ${ }^{5}$ Mourad Mekaouche, ${ }^{1,6}$ Jean-Michel Vallat, ${ }^{7}$ \\ Isabel IIla, ${ }^{3,4}$ and Jérôme J. Devaux ${ }^{1,5}$ \\ 'Aix Marseille Université, CNRS, CRN2M-UMR7286, Marseille, France. '2Université de Bordeaux, Interdisciplinary Institute for Neuroscience, UMR5297, Bordeaux, France. ${ }^{3}$ Neuromuscular Diseases \\ Unit, Hospital de la Santa Creu i Sant Pau, Universitat Autónoma de Barcelona, Barcelona, Spain. ${ }^{4}$ Centro para la Investigación en Red en Enfermedades Raras (CIBERER), Madrid, Spain. ${ }^{5}$ nnstitute for \\ Neurosciences of Montpellier, INSERM U1051, Montpellier University, Hopital Gui de Chauliac, Montpellier, France. ${ }^{6}$ Aix Marseille Université, CNRS, INP UMR7051, Marseille, France. `National Reference \\ Center for "rare peripheral neuropathies" and Department of Neurology, University Hospital, Limoges, France.
}

\begin{abstract}
Neurofascin-155 (Nfasc155) is an essential glial cell adhesion molecule expressed in paranodal septate-like junctions of peripheral and central myelinated axons. The genetic deletion of Nfasc155 results in the loss of septate-like junctions and in conduction slowing. In humans, IgG4 antibodies against Nfasc155 are implicated in the pathogenesis of chronic inflammatory demyelinating polyneuropathy (CIDP). These antibodies are associated with an aggressive onset, a refractoriness to intravenous immunoglobulin, and tremor of possible cerebellar origin. Here, we examined the pathogenic effects of patient-derived anti-Nfasc155 IgC4. These antibodies did not inhibit the ability of Nfasc155 to complex with its axonal partners contactin-1 and CASPR1 or induce target internalization. Passive transfer experiments revealed that IgC4 antibodies targeted Nfasc 155 on Schwann cell surfaces, and diminished Nfasc155 protein levels and prevented paranodal complex formation in neonatal animals. In adult animals, chronic intrathecal infusions of antibodies also induced the loss of Nfasc155 and of paranodal specialization and resulted in conduction alterations in motor nerves. These results indicate that anti-Nfasc155 IgG4 antibodies perturb conduction in the absence of demyelination, validating the existence of paranodopathy. These results also shed light on the mechanisms regulating protein insertion at paranodes.
\end{abstract}

\section{Introduction}

Chronic inflammatory demyelinating polyneuropathy (CIDP) is a syndrome affecting peripheral nerves and leading to significant disability $(1,2)$. Although it is widely accepted that this disorder has an autoimmune pathogenesis, the exact mechanisms leading to nerve conduction slowing or conduction loss are still elusive. Recent evidence indicates that autoantibodies targeting cell adhesion molecules at the nodes of Ranvier are implicated in CIDP pathogenesis in a subset of patients. Autoantibodies to contactin-1 (CNTN1), neurofascin-155 (Nfasc155), CNTN1-associated protein-1 (CASPR1), and neurofascin-186 (Nfasc186) have been described in subgroups of patients showing distinct clinical presentations (3-10). In the case of antibodies against Nfasc155, the clinical phenotype is clearly distinct from that of typical CIDP patients and includes a predominantly distal phenotype, a lowfrequency and high-amplitude tremor with cerebellar features (11), and a poor response to intravenous immunoglobulin (12). These cell adhesion molecules play important roles in nerve physiology by enabling the formation and stability of the voltage-gated

Conflict of interest: JJD received a research grant from CSL Behring. II holds a patent for dysferlin detection in monocytes (US20030165937A1), and has consulted for Grifols, Genzyme, Alexion, and UCB.

Copyright: (5) 2019, American Society for Clinical Investigation.

Submitted: September 7, 2018; Accepted: March 12, 2019; Published: April 29, 2019.

Reference information: J Clin Invest. 2019;129(6):2222-2236.

https://doi.org/10.1172/JCI124694. sodium channel $\left(\mathrm{Na}_{\mathrm{v}}\right)$ clusters at the nodes of Ranvier where the action potentials are regenerated $(13,14)$. It was thus speculated that these autoantibodies may affect conduction by altering the axoglial contacts, axonal physiology, or myelin insulation. Indeed, Nfasc186 is expressed at the nodal axolemma, where it interacts with gliomedin and NrCAM, 2 glial cell adhesion molecules. This complex is crucial for the initial clustering of $\mathrm{Na}_{\mathrm{v}}$ channels at heminodes (15), but also seems important for the stabilization of $\mathrm{Na}_{\mathrm{v}}$ channels at adult nodes of Ranvier and of microvilli structure surrounding the nodes of Ranvier (16-18). Recent evidence indicates that autoantibodies to Nfasc186 are associated with morphological alterations of the microvilli in sural biopsies from CIDP patients and the presence of reversible proximal conduction block (19). This suggests that anti-Nfasc186 antibodies may affect node function and lead to conduction failure. However, the exact mechanisms of how these morphological alterations lead to functional deficits remain to be demonstrated.

At paranodal regions, the association of the axonal protein CNTN1 and CASPR1 with the glial protein Nfasc155 forms an axoglial complex that is crucial for the formation of atypical adhesive junctions named septate-like junctions (20-22). These junctions spiral around the axons along the paranodes and help myelin insulation by decreasing the extracellular space between the paranodal myelin loop and the axon. The presence of autoantibodies against CNTN1, Nfasc155, or CASPR1 was found to be associated with alterations of the paranodal complex CNTN1/CASPR1/Nfasc155 and loss of septate-like junctions in biopsies from CIDP patients 
$(5,6,23-25)$. These autoantibodies are predominantly of the IgG4 isotype and are suspected to interfere with the function or structure of their target antigens to exert their deleterious effects (26, 27). Anti-CNTN1 IgG4 antibodies were recently shown to abolish the interaction between CNTN1/CASPR1 and Nfasc155 (28) and to penetrate the paranodal regions (29). Particularly, chronic exposure to these antibodies induces the loss of paranodal specialization and conduction (29). The mechanisms of how anti-Nfasc155 IgG4 antibodies affect conduction are, by contrast, yet unknown.

Here, we purified anti-Nfasc155 IgG4 antibodies from CIDP patients and tested their pathogenic function in CNTN1/CASPR1/ Nfasc155 interaction and in animal models. We found that antiNfasc155 IgG4 antibodies act in a different manner compared with antibodies against CNTN1. These autoantibodies do not determine a functional blocking of $\mathrm{Nfasc} 155$, and do not penetrate the paranodal regions. Instead, these antibodies bind to Nfasc155 on the surface of Schwann cells, induce the selective depletion of Nfasc155 in the peripheral nerves, and thus preclude the formation of the paranodal axoglial junction. These data indicate that the pathogenic mechanisms leading to conduction abnormalities are complex and involve different immune processes.

\section{Results}

Anti-Nfasc155 IgG4 does not affect the interaction between CNTN1/ CASPR1 and Nfasc155. In order to test the pathogenic role of antiNfasc155 autoantibodies, IgG4 antibodies were purified using CaptureSelect affinity matrix from plasmapheresis fluids or sera of 3 CIDP patients presenting with high titers of anti-Nfasc155 IgG4 (Supplemental Figure 1; supplemental material available online with this article; https://doi.org/10.1172/JCI124694DS1). These patients presented with a severe CIDP with predominant distal weakness and refractoriness to intravenous immunoglobulin. Patients CIDP1 and CIDP2 also had disabling tremors. Patient CIDP3 also had CNS involvement (optic neuropathy and periventricular white matter lesions). The clinical presentation of patient CIDP1 has been previously reported (9). The blood samples from these patients showed a predominant IgG4 reactivity against human Nfasc155 (27), and IgG4 binding to paranodal regions from rat sciatic nerve fibers (Supplemental Figure 1). We thus focused this study on IgG4 isotype. As control, IgG4 antibodies were purified from healthy donor plasma. The purity of the IgG4 samples was confirmed by immunoblots (Supplemental Figure 2).

First, we examined the bioactivity and the specificity of the purified IgG4 samples against rat Nfasc155 and rat nervous tissues, as our goal was to test the pathogenic function of these antibodies in rodent models. We confirmed that these purified IgG4 fractions reacted against rat Nfasc155 by Western blot against rat brain protein samples (Supplemental Figure 2B), but also by immunostaining on rat Nfasc155-expressing human embryonic kidney 293 (HEK293) cells and on teased rat sciatic nerve fibers (Supplemental Figures 3 and 4). The reactivity of IgG4 fractions against rat brain samples, rat Nfasc155, or rat sciatic nerves was abrogated after elimination of anti-Nfasc155 antibodies by immunoadsorption against Nfasc155 (Supplemental Figures 2-4). This clearly indicated that the paranodal fixation was due to a specific reactivity against Nfasc155. In keeping, no reactivity was found against rat CASPR1/CNTN1 or rat Nfasc186 (Supplemental Figure 3).
We next investigated whether anti-Nfasc155 IgG4 may affect the interaction between Nfasc155 and its axonal partners CNTN1 and CASPR1 using a cell aggregation assay. For that purpose, HEK293 cells were transfected with mCherry-conjugated rat Nfasc155, or with CNTN1 and GFP-tagged CASPR1. Cells were then mixed together and incubated for 2 hours under gentle agitation to enable cell aggregate formation. The percentage of cell aggregates showing contacts between mCherry-expressing (red) and GFP-expressing (green) cells was then quantified, as well as the percentage of green cells per aggregate. As negative controls, Nfasc155-expressing cells were incubated with cells expressing GFP alone. Under such conditions, minimal interaction was observed between Nfasc155- and GFP-expressing cells (Figure 1), and most cell aggregates contained either only Nfasc155expressing cells or only GFP-expressing cells. By contrast, Nfasc155-expressing cells formed numerous interactions with CNTN1/CASPR1-expressing cells, and most cell aggregates contained $50 \%$ green and red cells (Figure 1). To test the efficacy of anti-Nfasc155 IgG4 to dismantle these aggregates, $10 \mu \mathrm{g}$ of IgG4 was added to the cell mixture during the 2-hour incubation step. For comparison, anti-CNTN1 IgG4 antibodies were also tested. As previously described (28), anti-CNTN1 IgG4 potently inhibited the interaction of Nfasc155-expressing cells with CNTN1/ CASPR1. By contrast, anti-Nfasc155 IgG4 from the 3 distinct CIDP patients did not decrease cell-cell interaction (Figure 1). Interestingly, cell aggregates incubated with anti-Nfasc155 IgG4 presented a higher percentage of Nfasc155-expressing cells. This indicated that anti-Nfasc155 IgG4 antibodies do not prevent the interaction between Nfasc155 and its axonal partners CNTN1/ CASPR1, but may favor Nfasc155 clustering.

Anti-Nfasc155 IgG4 binds to surface antigens on Schwann cells. We previously reported that the function-blocking activity of anti-CNTN1 IgG4 enables these autoantibodies to dismantle the axoglial interaction and to penetrate the paranodal barrier (29). To determine whether anti-Nfasc155 IgG4 antibodies have a similar pathogenic potential, rat sciatic nerve segments were incubated in vitro for 1 hour with $10 \mu \mathrm{g}$ of anti-Nfasc155 IgG4 purified from the 3 distinct CIDP patients (Supplemental Figure 5). An important IgG4 deposition was observed around nodes of Ranvier and at the surface of Schwann cells with all samples (Supplemental Figure 5). However, no IgG4 deposition was found at paranodes even after 3 hours of incubation, and no alterations of the paranodal regions were observed. Incubation with $10 \mu \mathrm{g}$ of control IgG4 did not generate any staining (data not shown). To confirm these findings, intraneural injections of $10 \mu \mathrm{g}$ of antibodies were performed in the sciatic nerves of 1-month-old rats. IgG4 deposition was then monitored 1 and 3 days after the injection, and the sciatic nerve fibers were immunostained for $\mathrm{Na}_{\mathrm{v}}$ channels and CNTN1 to label nodes and paranodes, or for $\beta$-catenin to label the adherens junctions in noncompact myelin. Again, no IgG4 deposition was detected at paranodes at 1 or 3 days after injection, and no alterations of the paranodal regions were observed. Instead, an important IgG4 was seen along the adherens junctions on the surface of the Schwann cells at both 1 and 3 days after injection (arrows in Figure 2, A-C). In addition, an important IgG4 deposition was observed around the nodes of Ranvier (Figure 2, D and E). Confocal microscopy examination demonstrated that the IgG4 deposition did not colo- 
A
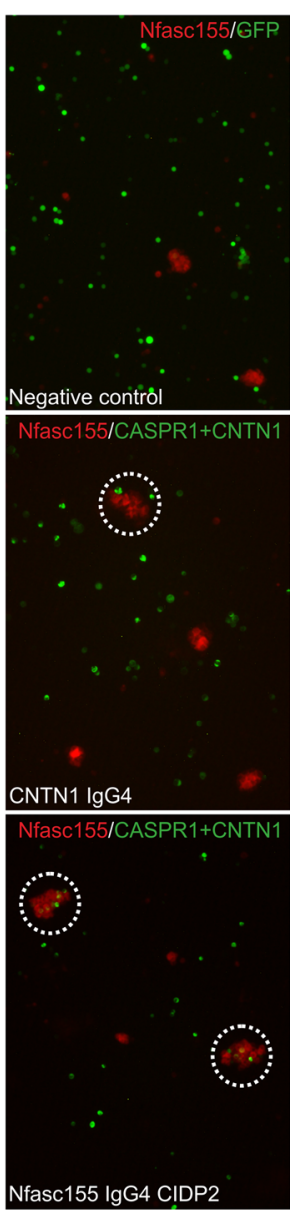

Nfasc155 lgG4 CIDP2
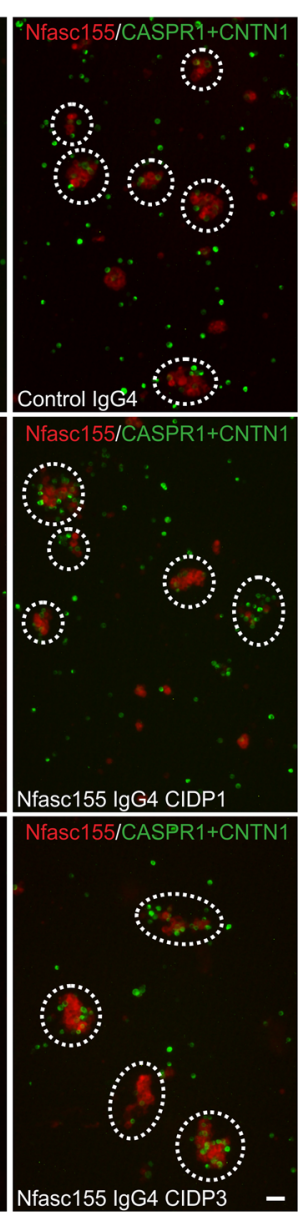

B

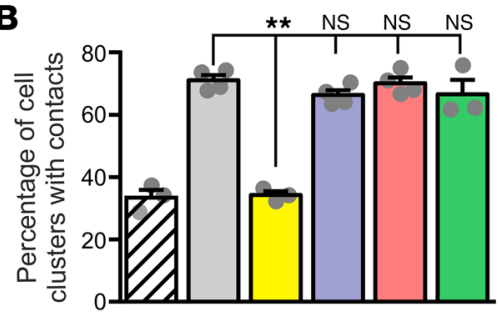

Megative control

$\square$ Control lgG4

口CNTN1 IgG4

Nasc155 IgG4 CIDP1

Nasc155 lgG4 CIDP2

ㅁfasc155 lgG4 CIDP3

\section{C}
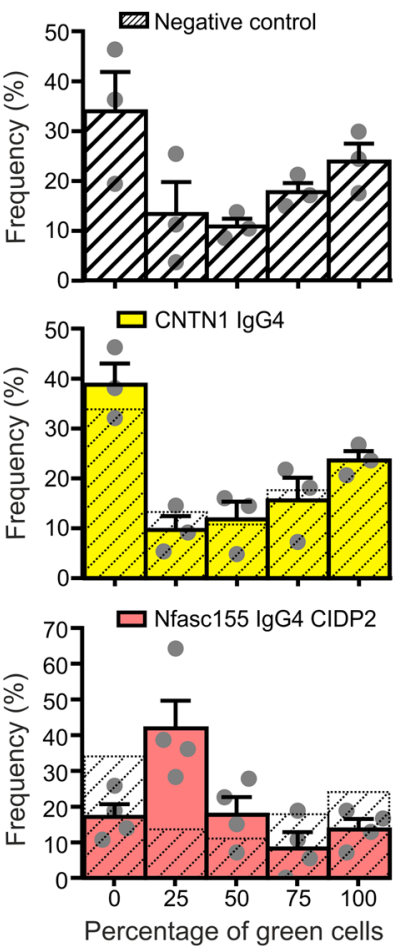

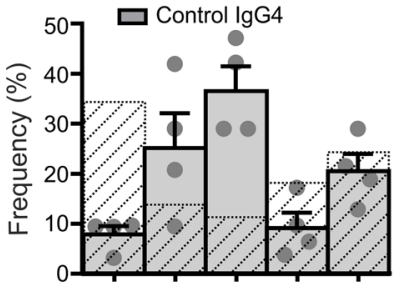

$\square$ Nfasc155 lgG4 CIDP1
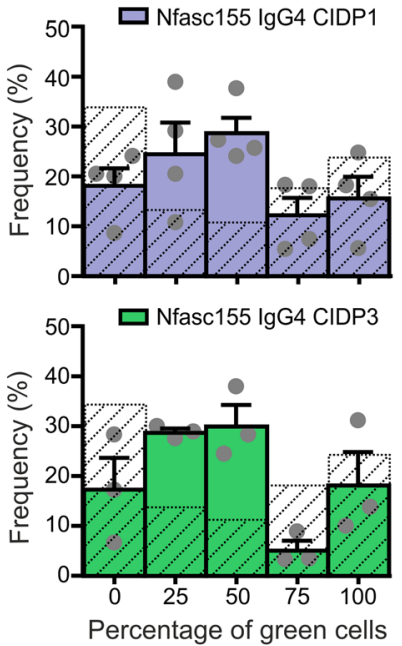

Figure 1. Antibodies to Nfasc155 do not alter the interaction between Nfasc155 and its axonal partners CNTN1 and CASPR1. (A-C) For aggregation assays, HEK293 cells were cotransfected with mCherry-tagged Nfasc155 or with GFP-tagged CASPR1 and CNTN1. Cells were then incubated under gentle agitation for 2 hours in the presence of $10 \mu \mathrm{g}$ of control IgG4, anti-CNTN1 IgG4, or anti-Nfasc155 IgC4 from 3 patients (CIDP1-3). As negative controls, Nfasc155-expressing HEK293 cells were incubated with cells expressing GFP alone (top left panel). Cells were examined with a fluorescence microscope at $\times 10$ objective. Representative fields are shown in $\mathbf{A}(n=3-4$ experiments for each condition). Dashed circles highlight cell aggregates with contacts between red and green cells. The percentage of cell clusters with contacts between green and red cells was quantified (B), as well as the relative frequency of green cells per aggregate (C) ( $n=3-4$ experiments for each condition). CASPR1/CNTN1- and Nfasc155-expressing cells form clusters. Anti-CNTN1 IgG4 significantly prevented the formation of cell aggregates ( ${ }^{*} P<0.005$ by unpaired 2 -tailed Student's $t$ tests for 2 samples of equal variance and by 1 -way ANOVA followed by Bonferroni's post hoc tests). By contrast, anti-Nfasc155 IgG4 did not affect the interaction between Nfasc155 and CASPR1/CNTN1. Bars represent mean and SEM. Scale bar: $50 \mu \mathrm{m}$.

calize with $\mathrm{Na}_{\mathrm{v}}$ channels at nodal axolemma, but may be located on Schwann cell microvilli surrounding the nodes. IgG4 deposition appeared in a disc-shaped manner around the nodes by 1 day, and became juxtanodal by 3 days after injection (Figure 2, D and E).

To confirm that this staining pattern was caused by the antiNfasc155 activity, we incubated sciatic nerve segments with IgG4 fractions immunoadsorbed against Nfasc155. In the 3 samples, the immunoadsorption completely abrogated the surface staining at nodes and adherens junctions (Supplemental Figure 5). In order to further determine whether this staining pattern corresponded to surface-bound antibodies or to internalized antibody/antigen complexes, we performed an acid wash following incubation with the anti-Nfasc155 IgG4 (Supplemental Figure 6). The acid wash completely removed the IgG4 deposition, indicating that IgG4 antibodies were bound to surface antigens. In a previous study, we found that anti-Nfasc155 IgG4 can also bind to Nfasc155 expressed on the surface of cultured oligodendrocytes (4). The acid wash also completely removed IgG4 deposition from the surface of cultured oligodendrocytes (Supplemental Figure 7), further indicating that anti-Nfasc155 IgG4 antibodies do not induce antigen internalization. Surface-bound antibodies fully colocalized with Nfasc155 after permeabilization in cultured rat oligodendrocytes (Supplemental Figure 7). Altogether, these results indicated that antibodies to Nfasc155 do not penetrate the paranodal domains, but bind to Nfasc155 expressed on the glial surface notably at adherens junctions and at the node vicinity.

Anti-Nfasc155 IgG4 depletes Nfasc155 and diminishes paranode formation. To determine whether anti-Nfasc155 antibodies may impair paranode formation/maintenance, we performed i.p. injections of anti-Nfasc155 IgG4 in neonatal animals before and 

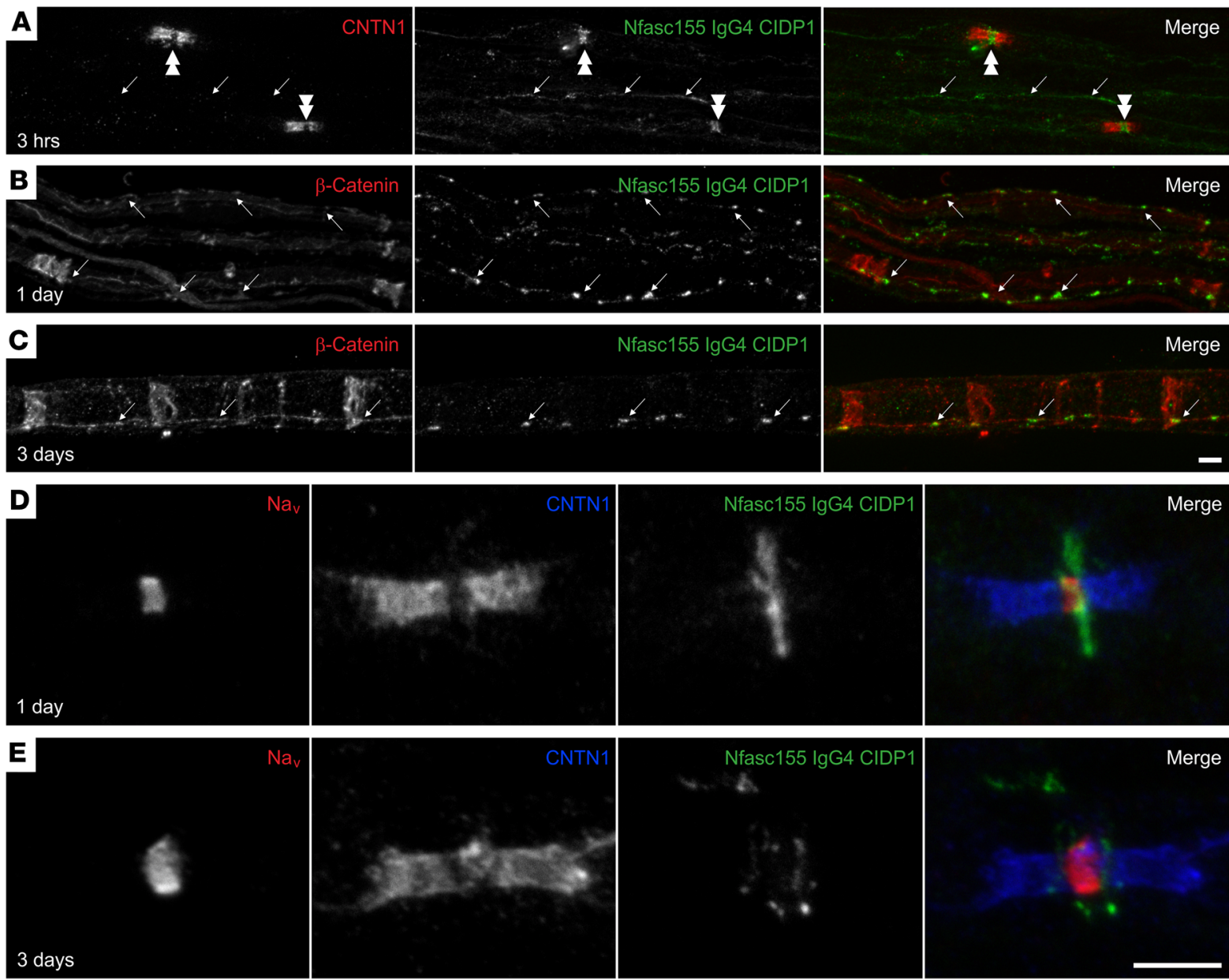

Figure 2. Anti-Nfasc155 autoantibodies target surface Schwann cell antigens. (A) Sciatic nerve fibers were incubated in vitro with purified anti-Nfasc155 IgG4 from patient CIDP1 for 3 hours, and immunolabeled for IgG4 (green) and CNTN1 (red). (B-E) Sciatic nerves were fixed 1 day (B and D) or 3 days (C and E) after intraneural injections of anti-Nfasc155 lgG4, and immunolabeled for lgG4 (green) and $\beta$-catenin (red; B and C) or CNTN1 (red; $\mathbf{D}$ and E) and Na channels (blue; $\mathbf{D}$ and E). Note that anti-Nfasc155 IgG4 bound to the surface of the Schwann cells and deposited at the vicinity of the node of Ranvier (double arrowheads) and at adherens junctions along the internode stained here with $\beta$-catenin (arrows). However, no penetration across the paranodal region was observed (images are representative of $n=3$ independent experiments). Scale bars: $10 \mu \mathrm{m}$.

after paranode formation. In the PNS, it has been reported that the aggregation of Nfasc186 or gliomedin at heminodes bordering the Schwann cells precedes that of Nfasc155 and CASPR1 at paranodes $(30,31)$. In keeping, we found that at postnatal day $\mathrm{O}$ (PO), most $\mathrm{Na}_{\mathrm{v}}$ channel aggregates lacked CASPR1 immunoreactivity at their borders (Figure 3). However, by P2 and P4, more than $90 \%$ of the $\mathrm{Na}_{\mathrm{v}}$ channel aggregates were flanked by CASPR1-positive paranodes. At these ages, the blood-nerve barrier is not functional and remains permeable until P13 (32). We thus performed injections of anti-Nfasc155 IgG4 $(100 \mu \mathrm{g})$ at P0 and $\mathrm{P} 4$; then the animals were sacrificed 2, 4, and 6 days later. As control, animals received similar doses of control IgG4. The percentages of $\mathrm{Na}_{\mathrm{v}}$ channel clusters with 1, 2, or no CASPR1positive paranodes were then quantified at all ages tested (Figure 3 and Supplemental Figure 9). Because the amount of IgG4 purified from patients CIDP2 and CIDP3 was limiting, we centered this study on the IgG4 fraction from patient CIDP1. In accordance with our hypothesis, the injection of anti-Nfasc155 IgG4 from patient CIDP1 at PO strongly impaired paranode forma- tion, and $29 \%$ of the $\mathrm{Na}_{\mathrm{v}}$ channel clusters lacked paranodes by $\mathrm{P} 2$ (vs. $6 \%$ in control animals). As a result, the percentage of nodes flanked by 2 CASPR1-positive paranodes was drastically diminished at this age (35\% vs. $66 \%$ in control animals). The remaining CASPR1-positive paranodes were shorter in comparison with control animals (Figure 3D). By P4, the percentage of nodes and heminodes reached normal values and was not significantly different between Nfasc155 IgG4 and control groups. Nonetheless, the paranodal length was still significantly shorter in comparison with control animals. By P6, no significant differences were seen between Nfasc155 IgG4 and control groups. When the injection of anti-Nfasc155 IgG4 was performed at P4, no significant effects were observed on paranodal length or paranode formation either 2 or 6 days after injections (Supplemental Figure 9). To confirm these findings, anti-Nfasc155 IgG4 antibodies from patients CIDP2 and CIDP3 were injected at PO, and the effects on paranodal formation at P2 were monitored (Supplemental Figure $10, \mathrm{~A}-\mathrm{E})$. In keeping with the previous findings, the injection of anti-Nfasc155 IgG4 from patients CIDP2 and CIDP3 potently 

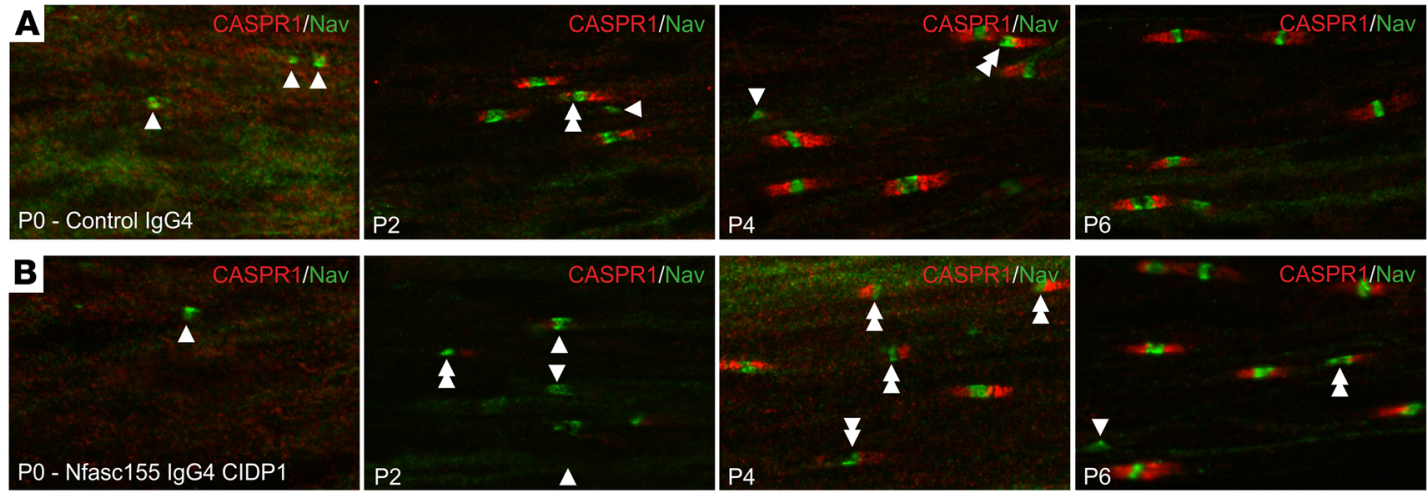

C
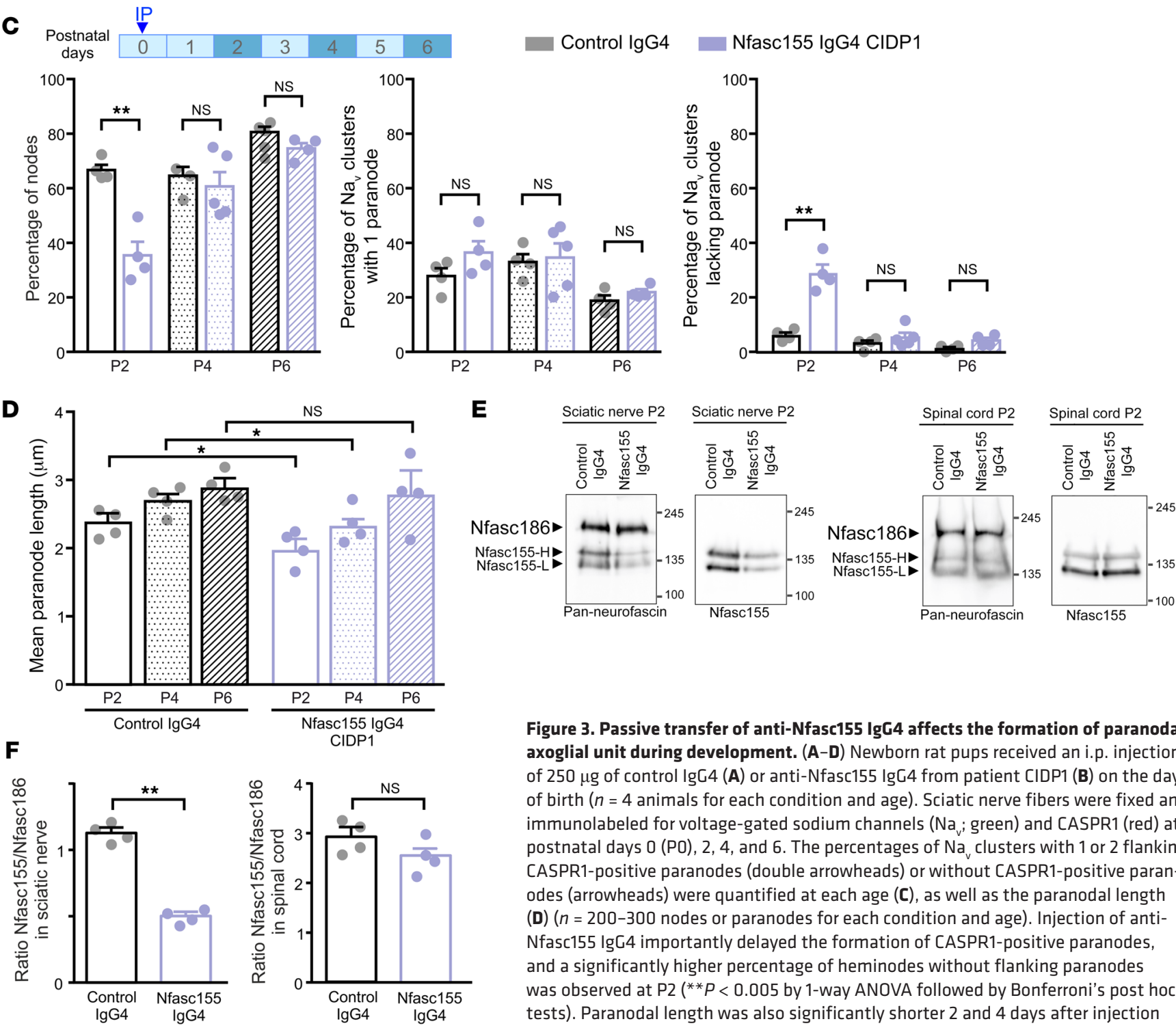

Figure 3. Passive transfer of anti-Nfasc155 IgG4 affects the formation of paranodal axoglial unit during development. (A-D) Newborn rat pups received an i.p. injection of $250 \mu \mathrm{g}$ of control IgG4 (A) or anti-Nfasc155 IgG4 from patient CIDP1 (B) on the day of birth ( $n=4$ animals for each condition and age). Sciatic nerve fibers were fixed and immunolabeled for voltage-gated sodium channels ( $\mathrm{Na}_{\mathrm{v}}$; green) and CASPR1 (red) at postnatal days $0(\mathrm{PO}), 2,4$, and 6 . The percentages of $\mathrm{Na}_{v}$ clusters with 1 or 2 flanking CASPR1-positive paranodes (double arrowheads) or without CASPR1-positive paranodes (arrowheads) were quantified at each age (C), as well as the paranodal length (D) ( $n=200-300$ nodes or paranodes for each condition and age). Injection of antiNfasc155 IgG4 importantly delayed the formation of CASPR1-positive paranodes, and a significantly higher percentage of heminodes without flanking paranodes was observed at $\mathrm{P} 2$ ( ${ }^{* *} P<0.005$ by 1 -way ANOVA followed by Bonferroni's post hoc tests). Paranodal length was also significantly shorter 2 and 4 days after injection of anti-Nfasc155 IgG4 ( ${ }^{*} P<0.05$ by 1-way ANOVA followed by Bonferroni's post hoc tests). Scale bar: $10 \mu \mathrm{m}$. (E and F) Sciatic nerve and spinal cord proteins (100 $\mu \mathrm{g})$ from P2 animals injected with control lgG4 $(n=4)$ or anti-Nfasc155 IgG4 $(n=4)$ at P0 were immunoblotted with antibodies recognizing all neurofascin isoforms (Pan-neurofascin) or specifically Nfasc 155 . The level of Nfasc155 was quantified relatively to that of Nfasc186 in both sciatic nerve and spinal cord (F). Nfasc155 level was significantly decreased in sciatic nerves of animals treated with anti-Nfasc155 lgC4, but not in spinal cord ( ${ }^{* *} P<0.005$ by unpaired 2 -tailed Student's $t$ tests for 2 samples of equal variance). Molecular weight markers are shown on the right (in kilodaltons). Bars represent mean and SEM. 

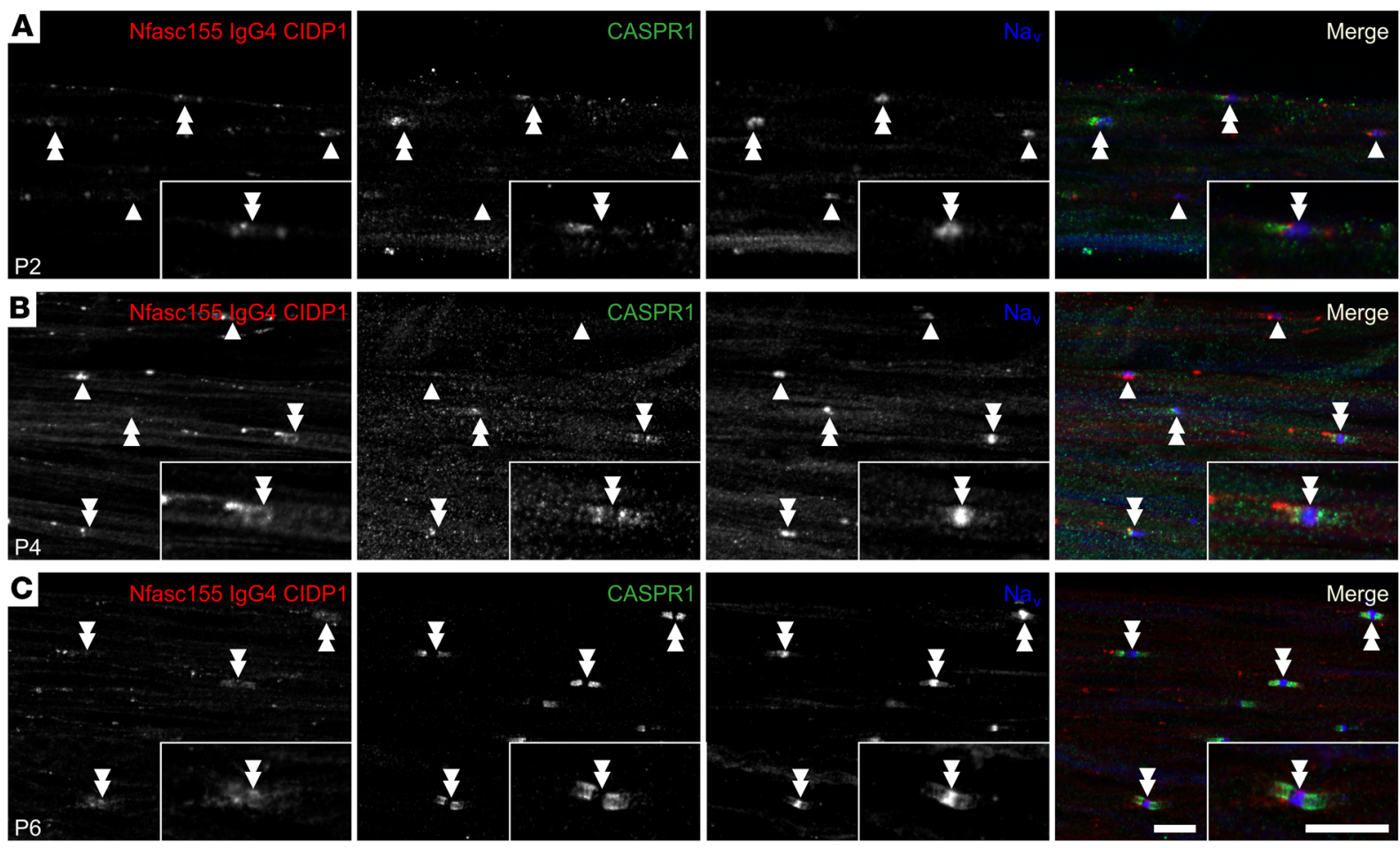

Figure 4. Anti-Nfasc155 IgG4 deposits at proximity of nodes in neonatal animals. (A-C) Newborn rat pups received daily i.p. injection of $250 \mu \mathrm{g}$ of control IgG4 (A) or anti-Nfasc155 IgG4 from patient CIDP1 (B) from the day of birth to P6 (images are representative of $n=3$ independent experiments for each age). Sciatic nerve fibers were immunolabeled for voltage-gated sodium channels ( $\mathrm{Na}_{\mathrm{v}}$; blue), CASPR1 (green), and IgC (red) at P2 (A), P4 (B), and P6 (C). At P2 and P4, IgG deposits were mostly detected at the borders of $\mathrm{Na}_{\mathrm{v}}$ clusters, even in $\mathrm{Na}_{\mathrm{v}}$ clusters lacking CASPR1-positive paranodes (arrowheads). No colocalization with CASPR1 was found even in $\mathrm{Na}_{v}$ clusters flanked by 1 or 2 paranodes (double arrowheads). At P6, IgC deposits appeared fainter and were mostly detected at the node vicinity. A weak IgC labeling can be observed at paranodes in some fibers. Isolated node labeling is shown at a higher magnification in the insets. Scale bars: $10 \mu \mathrm{m}$.

impaired paranode formation, and resulted in a strong decrease of CASPR1-positive paranode length (Supplemental Figure 10E).

To determine whether paranode formation impairment is linked to paranodal protein depletion or hypomyelination, sciatic nerve proteins were extracted at P2 and immunoblotted for neurofascin isoforms, CASPR1, E-cadherin, and neurofilament-200 (Figure 3 and Supplemental Figure 8). In addition, myelination was visualized on semithin nerve cross sections at P2 (Supplemental Figure 8). No overt myelination defects were observed in Nfasc155 IgG4-treated pups on semithin sections. Also, levels of E-cadherin and neurofilament-200 were normal. The levels of neurofascin isoforms were quantified using pan-neurofascin antibodies that recognize both Nfasc186 and the 2 forms of Nfasc155, Nfasc155 high (Nfasc155-H) and low (Nfasc155-L)(33). Nfasc155 level was monitored using an antibody specific to Nfasc155 recognizing both forms (Figure 3). The level of Nfasc186 was unchanged in both control and Nfasc155 IgG4 groups; however, the Nfasc155 level was significantly decreased in the sciatic nerves of anti-Nfasc155 IgG4-treated pups. CASPR1 level appeared normal in the sciatic nerves of the Nfasc155 IgG4 group (Supplemental Figure 8), suggesting that Nfasc155 depletion does not impact the level of the other paranodal proteins. Also, Nfasc155 level was unchanged in the spinal cord, presumably because the blood-brain barrier, which is functional at birth, may have precluded antibody diffusion into the CNS.
The level of Nfasc155 was also examined in pups treated with anti-Nfasc155 IgG4 at P4 and sacrificed at P6. No significant differences were seen between control and Nfasc155 IgG4 groups (Supplemental Figure 9). The late injection of autoantibodies at P4 thus did not appear to affect paranode stability or Nfasc155 level. This indicated that anti-Nfasc155 IgG4 antibodies do not impact Nfasc155 particles that are stably incorporated at paranodes, but induce their elimination before their stabilization at paranodes. To further confirm this hypothesis, daily i.p. injections of anti-Nfasc155 IgG4 were performed from P0 to P6, and paranode formation was examined at P4 and P6 (Supplemental Figure 10, F-K). The extension of the infusion duration resulted in a significant impairment of paranode formation at P4 and in a significant shortening of paranodal length (Supplemental Figure 10K). By P6, the percentage of $\mathrm{Na}_{\mathrm{v}}$ clusters flanked by 1 or 2 CASPR1-positive paranodes and the paranodal length were not significantly different from those in control animals. This indicated that a longer antibody infusion time further delayed paranode formation, but could not counteract the massive myelin formation process taking place at P6. In order to determine whether these alterations are associated with a loss of septate-like junctions, an electron microscopic observation was done at $\mathrm{P} 4$ when the effects of autoantibodies are important. Despite the young age of the animals, many nodes of Ranvier were observed in both groups of animals. In control animals, numerous septate-like junctions 
A

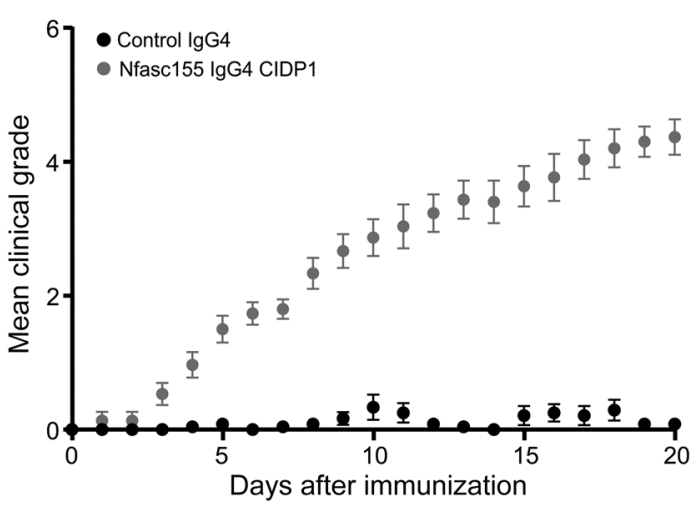

B

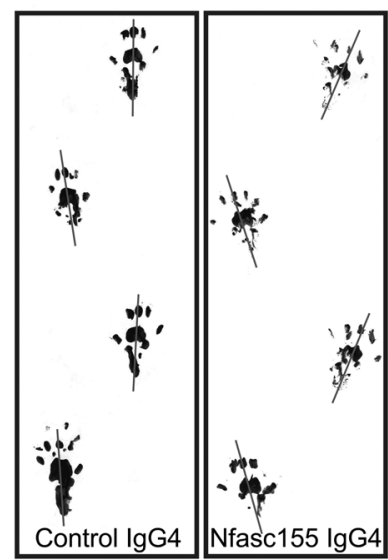

C

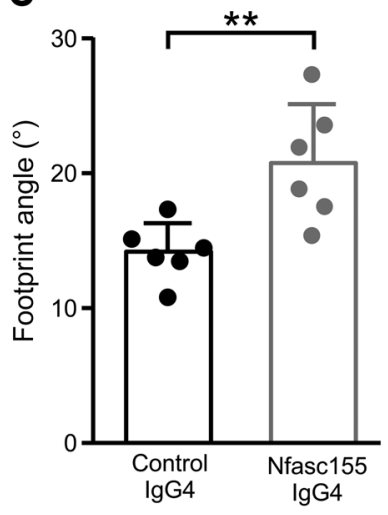

D

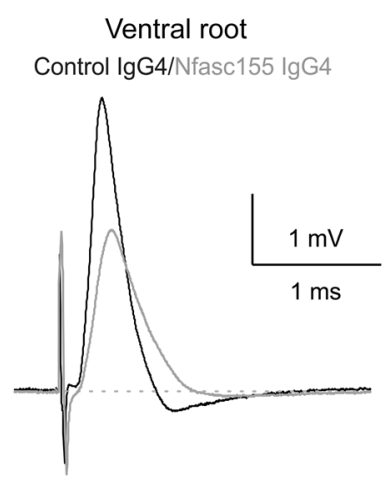

$\mathbf{F}$

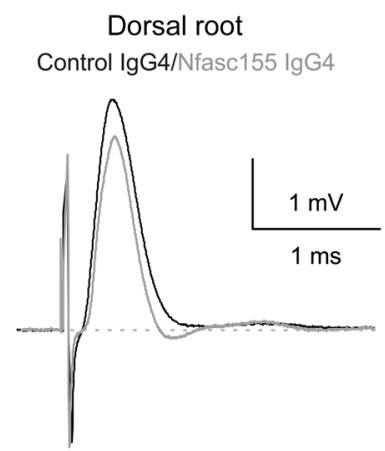

E

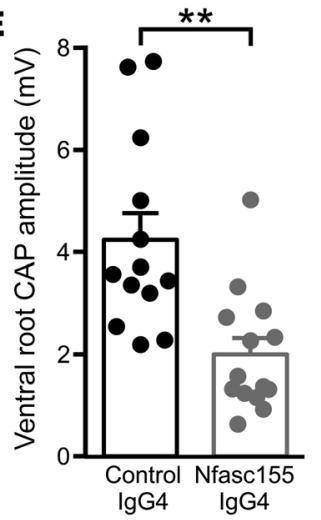

G

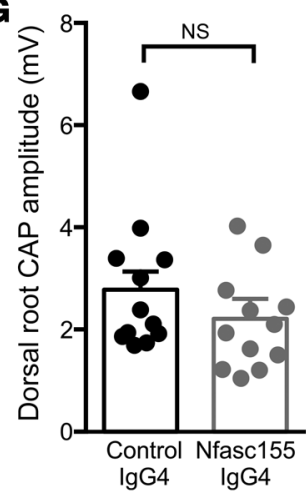

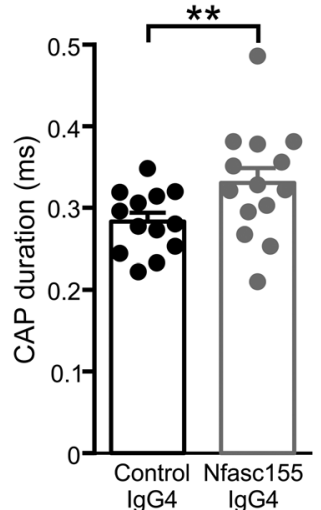
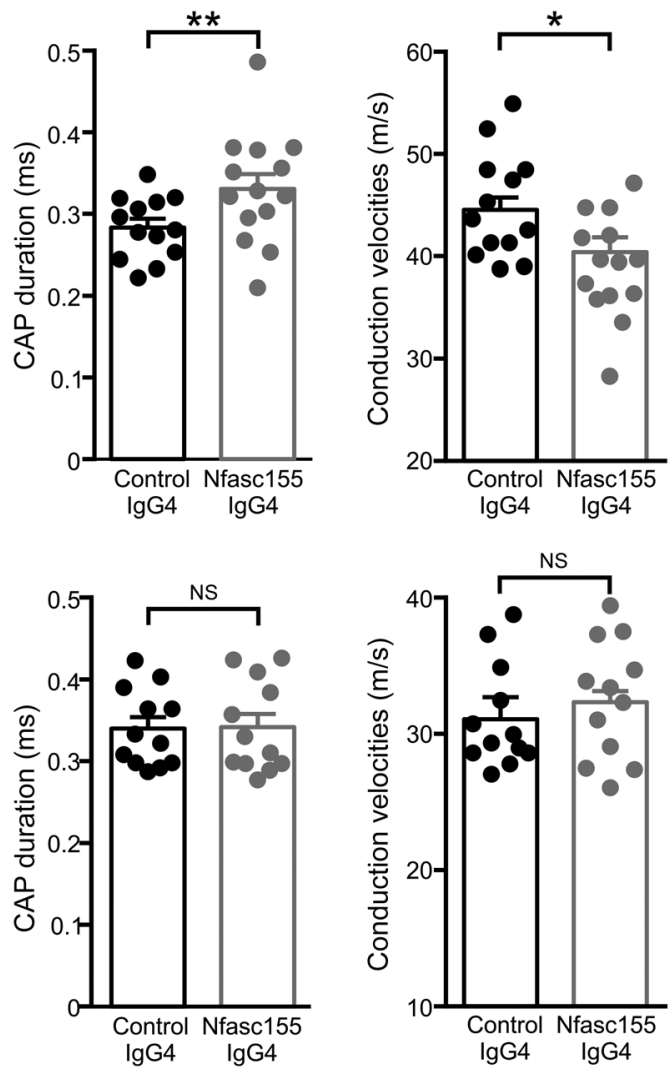

Figure 5. The chronic intrathecal infusion of autoantibodies induces gait abnormalities and motor nerve conduction slowing. (A-C) Adult Lewis rats received daily intrathecal infusions (100 $\mu \mathrm{g} / \mathrm{d}$ ) of control lgG4 (black circles; $n=15$ animals) or anti-Nfasc155 IgG4 from patient CIDP1 (gray circles; $n=15$ animals) during 3 weeks. The clinical score was monitored daily and averaged. The passive infusion of anti-Nfasc155 IgC4 induced progressive clinical symptoms. (B and C) Footprint analysis revealed abnormal spreading of hind limbs in animals treated with anti-Nfasc155 IgG4 compared with control animals. The footprint angle (gray lines) was significantly increased in animals treated with anti-Nfasc155 IgG4 compared with controls. (D-G) L6 ventral and dorsal roots from animals injected with control lgG4 or anti-Nfasc155 IgG4 were recorded on day 21 after the beginning of the injections ( $n=12-14$ nerves from 12-14 animals). Representative CAPs from control IgG4-treated (black traces) and anti-Nfasc155 IgG4-treated (gray traces) animals are shown in D and $\mathbf{F}$. The peak amplitude, CAP duration, and conduction velocities at peak amplitude are represented in $\mathbf{E}$ and $\mathbf{G}$ for ventral and dorsal roots, respectively. The ventral spinal nerves of animals treated with anti-Nfasc155 IgG4 showed a significant decrease in CAP amplitude and conduction velocity compared with controls. This was associated with a significant increase in CAP duration. By contrast, nerve activity was not significantly affected in dorsal root. ${ }^{*} P<0.05,{ }^{*} P<0.005$ by unpaired 2 -tailed Student's $t$ tests for 2 samples of equal variance. Bars represent mean and SD.

were observed at paranodal loops bordering the nodes of Ranvier (Supplemental Figure 11). By contrast, animals treated with anti-Nfasc155 IgG4 presented important alterations at paranodal regions (Supplemental Figure 11). Some axons completely lacked septate-like junctions at paranodes, whereas others presented paranodal loops with fewer septate-like junctions (Supplemental Figure 11). However, no alterations of the microvilli contacting the nodes of Ranvier were found. Altogether, this indicated that the loss of CASPR1 labeling at paranodes was correlated with a disappearance of septate-like junctions. 

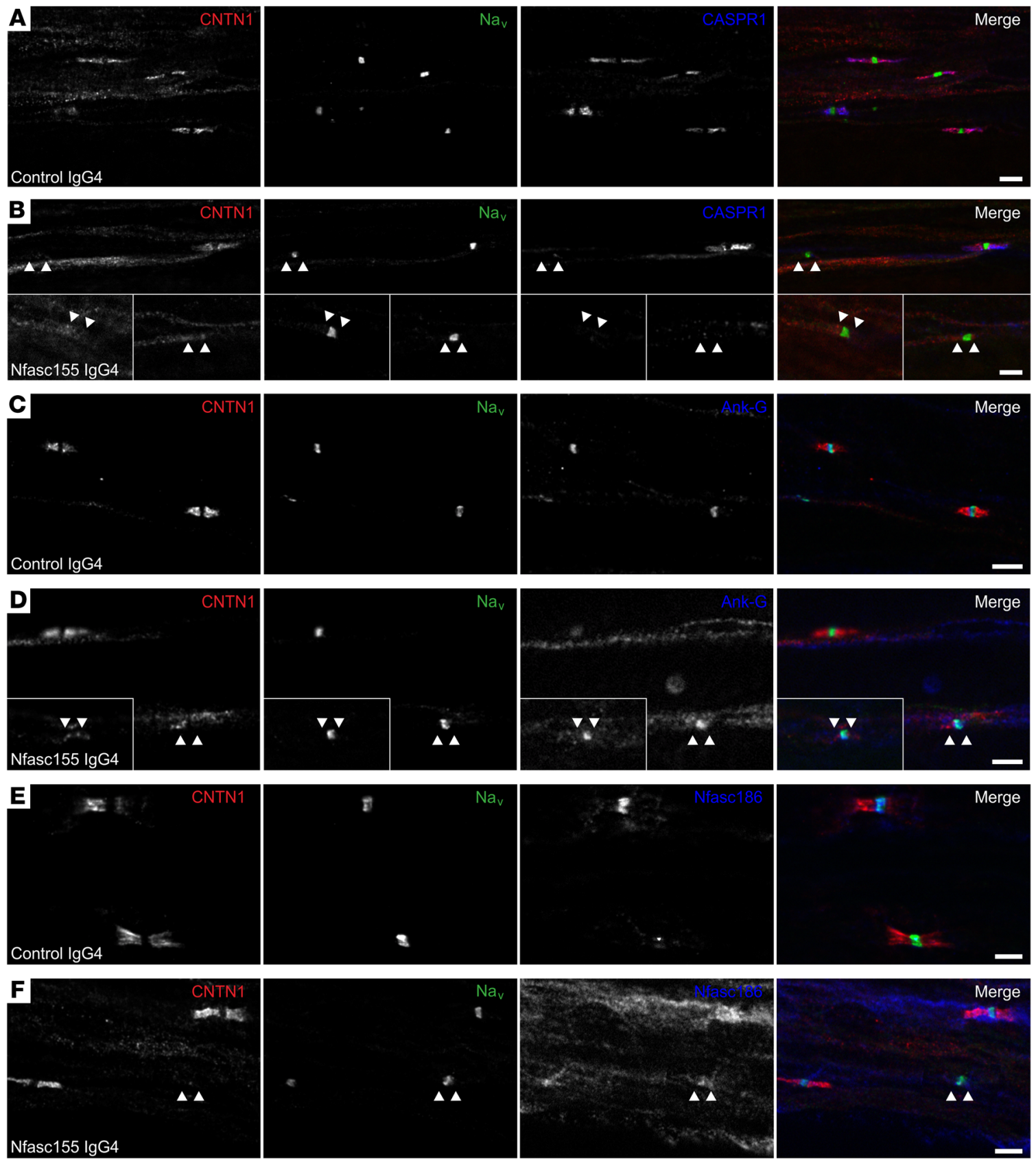

Figure 6. Anti-Nfasc155 IgG4 disrupts paranodal specialization in ventral spinal nerve. (A-F) Teased fibers from L6 ventral roots of animals treated with control IgG4 ( $n=15 ; \mathbf{A}, \mathbf{C}$, and E) or anti-Nfasc155 IgG4 from patient CIDP1 ( $n=15 ; \mathbf{B}, \mathbf{D}$, and F) were stained for Na channels (green), CNTN1 (red), and CASPR1 (blue, A and B), ankyrin-G (Ank-C; blue, C and D), or Nfasc186 (blue, $\mathbf{E}$ and F). Most nodes of Ranvier were positive for Na ${ }_{v}$ channel, ankyrin-G, and Nfasc186 clusters and were bordered by paranodes stained for CASPR1 and CNTN1 in control animals. By contrast, many nodes lacked CASPR1 or CNTN1 staining at paranodes (arrowheads) in animals chronically injected with anti-Nfasc155 IgG4. Insets in B and D show representative affected nodes. Scale bars: $10 \mu \mathrm{m}$.

Because IgG4 deposition onto the Schwann cell surface was detected after intraneural injection in adult animals, IgG4 deposits were also investigated in sciatic nerves from P2, P4, and P6 animals (Figure 4). Strong IgG4 staining was observed at the border of $\mathrm{Na}_{\mathrm{v}}$ clusters at both P2 and P4 (Figure 4). However, no colocalization with CASPR1 was detected, and the regions stained for IgG4 were devoid of CASPR1 labeling. In most axons, IgG4 deposits flanked $\mathrm{Na}_{\mathrm{v}}$ channel clusters lack- ing CASPR1-positive paranodes, indicating that IgG4 deposition may precede paranode complex formation, and affect its formation. In some axons, the IgG4 deposits were intercalated in between CASPR1 and $\mathrm{Na}_{\mathrm{v}}$ channels (inset in Figure 4A). At P6, most $\mathrm{Na}_{\mathrm{v}}$ channel clusters were flanked by 2 paranodes, and the IgG4 deposition was restricted to the nodal vicinity (double arrowheads in Figure 4C). Many axons presented a faint IgG4 deposition at paranodes that colocalized with CASPR1 at this 
A
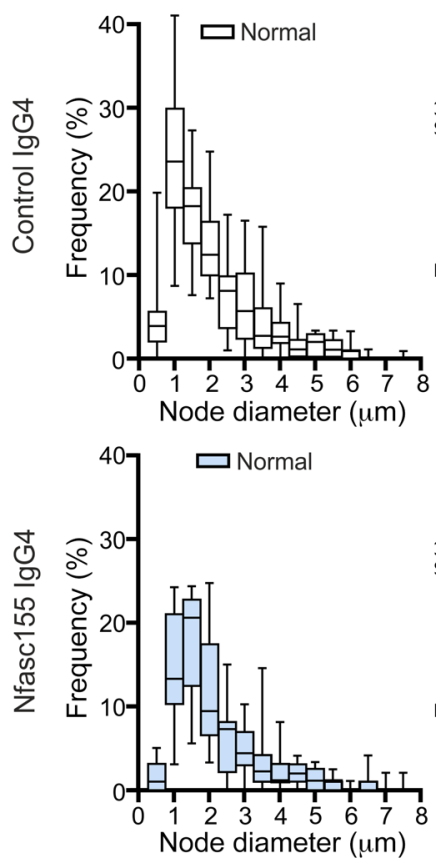

C
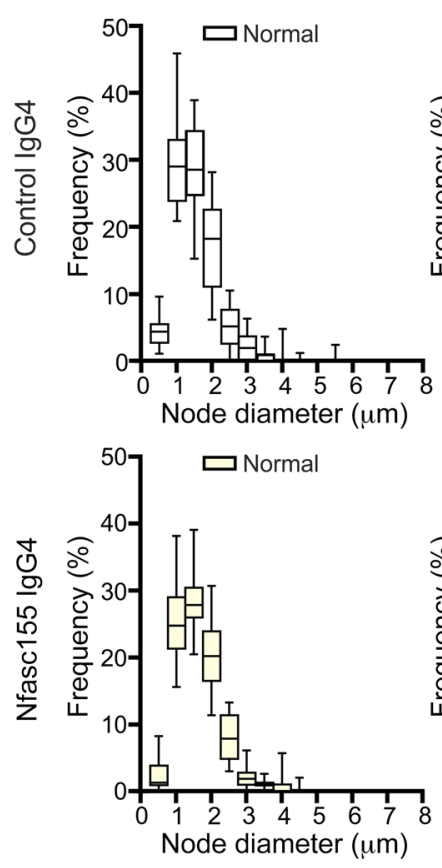

Ventral root
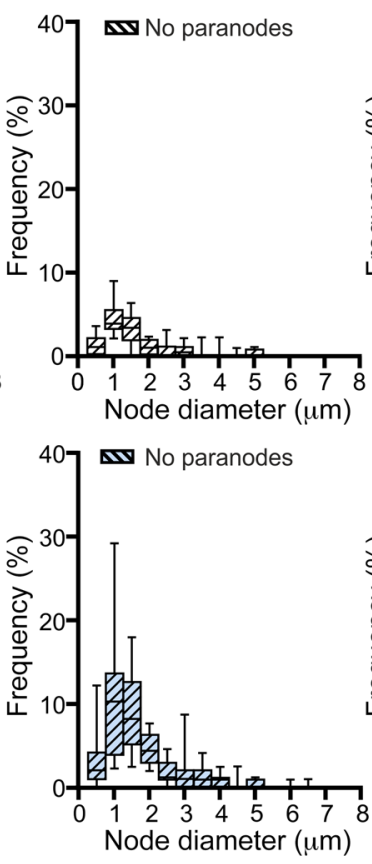

Dorsal root
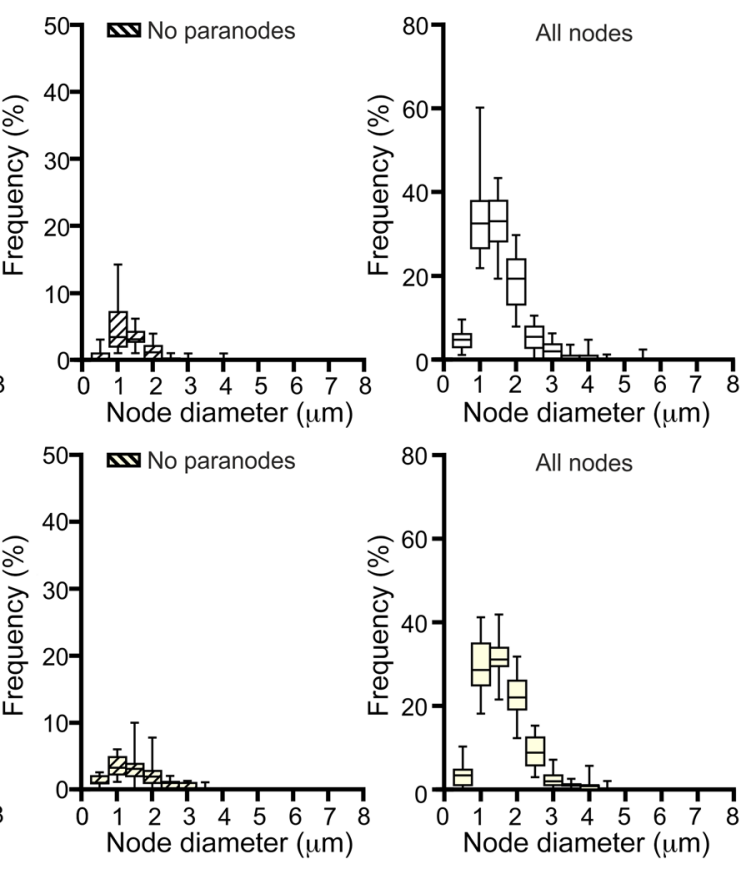

B
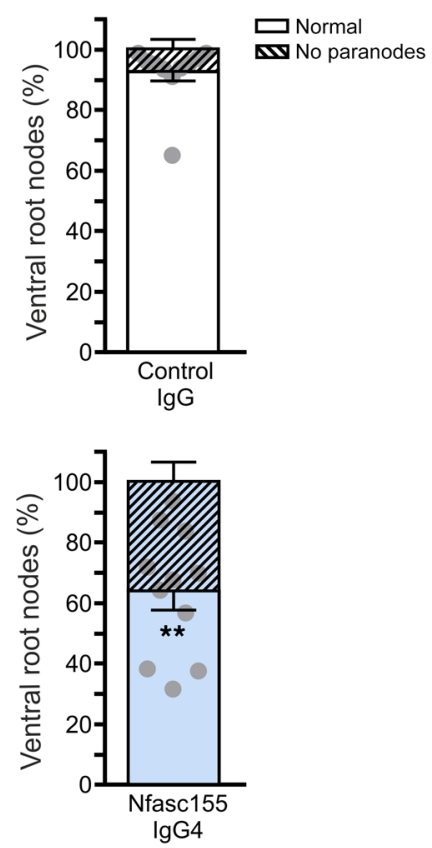

D
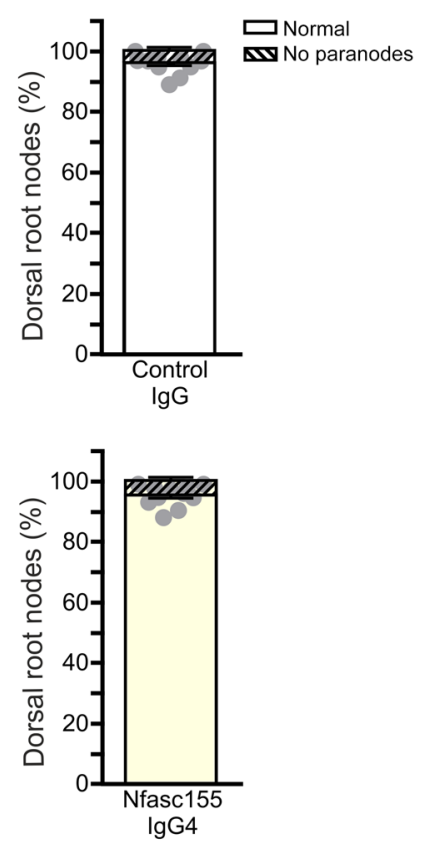

E

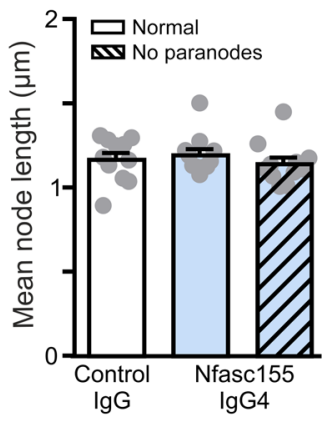

Ventral root

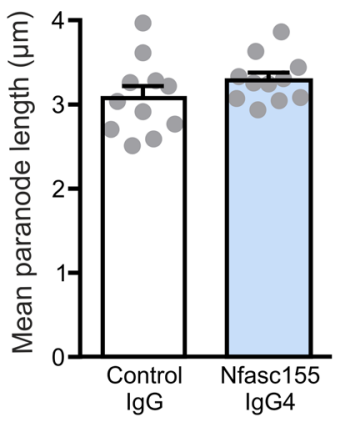

F

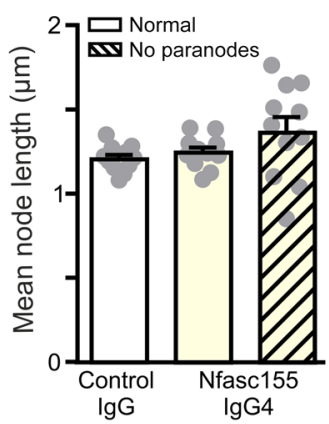

Dorsal root

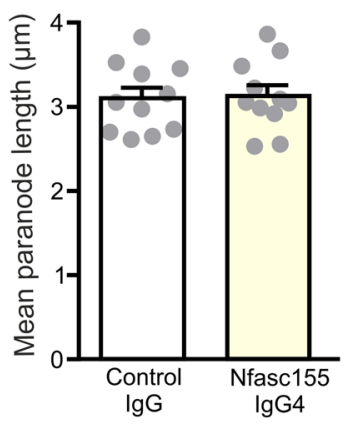


Figure 7. Autoantibodies preferentially affect motor axons. (A-D) The distribution of the axonal population according to the nodal diameters of axons was examined in L6 ventral roots ( $\mathbf{A}$ and $\mathbf{B}$ ) and dorsal roots ( $\mathbf{C}$ and D) of animals treated with control IgG4 ( $n=1297$ nodes for ventral roots and 1061 nodes for dorsal roots from 11 animals) or anti-Nfasc155 lgC4 from patient CIDP1 ( $n=1376$ nodes for ventral roots and 875 nodes for dorsal roots from 11 animals). The respective proportion of normal nodes (open boxes) or nodes lacking paranodes (hatched boxes) is represented on the left, and the distribution of the total axonal population is represented on the right. The percentage of nodes showing paranodal alterations was calculated in ventral (B) and dorsal roots (D). Scatter plots represent the percentage of normal nodes in each animal. Paranodes were more significantly affected in ventral root axons than in dorsal roots ( ${ }^{*} P<0.005$ by unpaired 2-tailed Student's $t$ tests for 2 samples of equal variance). ( $E$ and F) The mean length of nodes lacking paranodes (hatched boxes) or appearing normal (open boxes) was measured in 11 animals for each group, as well as the mean paranodal length. No significant alterations in node or paranode length were observed between control and anti-Nfasc155 IgC4-treated animals (unpaired 2-tailed Student's $t$ tests and 1-way ANOVA followed by Bonferroni's post hoc tests). In $\mathbf{B}$ and $\mathbf{D}-\mathbf{F}$, bars represents mean and SEM. In $\mathbf{A}$ and $\mathbf{C}$, box bounds represent the first and third quartiles, lines within the box represent the median, and whiskers show the minimal and maximal values.

age, suggesting that a proportion of the antibody/antigen complex was incorporated at paranodes.

The chronic infusion of autoantibodies induces paranodal dismantling and conduction alterations. The existence of protein turnover at paranodes has been demonstrated in a tamoxifeninducible Cre mouse line. In these animals, Nfasc155 levels were shown to decline 20 days after Nfasc gene ablation in Schwann cells (34). We thus explored whether long-term exposure to antiNfasc155 IgG4 may alter Nfasc155 levels in adult animals and affect paranode maintenance. For that purpose, chronic infusion of autoantibodies was implemented in naive animals. To bypass the blood-nerve barrier that can limit the diffusion of circulating antibodies to nerve fibers, an intrathecal catheter was implanted into the rachidian canal of Lewis rats to enable infusion of antibody in the cerebrospinal fluid (CSF) bathing the lumbar spinal roots. This procedure did not alter animal behavior or gait (Supplemental Video 1). Intrathecal infusion is relevant to CIDP pathology because anti-Nfasc155 IgG4 can be detected in the CSF and prominent nerve root involvement has been described in patients using magnetic resonance neurography $(35,36)$. Daily injections of anti-Nfasc155 IgG4 from patient CIDP1 or control IgG4 were performed for a period of 20 days. After 5 injections of anti-Nfasc155 IgG4, all animals developed tail weakness or paralysis (Figure 5). The clinical deficits then progressively worsened as treatment was pursued, and after 20 days, all animals presented a complete tail paralysis, an unsteady gait, and paraparesis (Figure 5 and Supplemental Video 1). In contrast, none of the 15 animals treated with the same amount of control IgG4 developed significant symptoms. Footprint analysis showed increased outward rotation of the hind paws in animals treated with anti-Nfasc155 IgG4 (Figure 5, B and C). The hindlimb grip strength test did not reveal significant differences between Nfasc155 and control IgG4 groups (data not shown).

Electrophysiological examination was performed at 21 days and revealed important abnormalities in ventral spinal roots
(Figure 5, D and E). Compound action potentials (CAPs) from ventral spinal roots of anti-Nfasc155 IgG4-treated animals had a decreased amplitude in comparison with control IgG4 animals (Figure 5). This decreased activity was associated with a significant slowing in nerve conduction velocity and a significant increase in CAP duration suggestive of temporal dispersion. CAP recruitment and refractory period were, however, not significantly impacted. By contrast to ventral roots, dorsal spinal nerves (Figure 5) or sciatic nerves (data not shown) did not exhibit conduction abnormalities. This indicated that anti-Nfasc155 IgG4 induced conduction slowing in motor nerves mimicking a demyelinating phenotype.

We then examined whether myelin alterations may be responsible for these conduction defects. No obvious signs of demyelination or node disorganization were observed in ventral and dorsal spinal nerves from animals treated with anti-Nfasc155 IgG4 (Figure 6 and Supplemental Figure 12). All nodes showed normal clusters of Nfasc186, $\mathrm{Na}_{v}$ channels, and ankyrin-G. However, CNTN1 and CASPR1 staining was often absent from paranodes in animals treated with anti-Nfasc155 IgG4 (Figure 6). These alterations were more prominent in the ventral spinal nerves from anti-Nfasc155 IgG4-treated animals $(36.1 \% \pm 6.3 \%$ vs. $7.4 \% \pm 3.1 \%$ in control IgG4; Figure 6) than in dorsal spinal nerves $(4.8 \% \pm 1.0 \%$ vs. $4.0 \%$ $\pm 1.0 \%$ in control IgG4; Figure 7 and Supplemental Figure 12).

Morphometric analysis did not reveal differences in nodal length between normal-appearing nodes and those lacking paranodes. The paranodal length was not significantly different between Nfasc155 IgG4 and control groups. Also, paranodal alterations were not associated with a specific subset of axons. These alterations were observed in both small- and large-diameter axons (Figure 7). The mean diameter of normal nodes and those lacking paranodes was not significantly different $(2.1 \pm 0.5 \mu \mathrm{m}$ vs. $1.7 \pm 0.4$ $\mu \mathrm{m}$, respectively; $n=15)$.

Anti-Nfasc155 IgG4 induces the selective depletion of Nfasc155. We investigated whether the loss of paranodal specialization may be associated with protein depletion. For that purpose, proteins were extracted from ventral and dorsal nerve roots and immunoblotted for Nfasc155 and CASPR1, but also E-cadherin and neurofilament-200 (Figure 8). Nfasc155 protein level was significantly decreased by nearly $40 \%$ in the ventral spinal nerves of animals treated with anti-Nfasc155 IgG4 compared with control animals, but was not altered in dorsal spinal nerves. These results matched the morphological alterations seen in ventral spinal nerves. No difference was observed in CASPR1 protein level in both ventral and dorsal spinal nerves in comparison with control animals. Similarly, the protein levels of neurofilament-200 and E-cadherin were unaffected. This was in keeping with the absence of axonal or myelin alterations in these animals.

\section{Discussion}

The principal finding of this study is that human anti-Nfasc155 IgG4 antibodies purified from CIDP patients induce clinical deficits and nerve conduction abnormalities resembling those of CIDP patients with anti-Nfasc155 antibodies. We provide evidence that these autoantibodies bind to the glial surface and cause Nfasc155 depletion and affect the formation of paranodal axoglial specialization. Interestingly, our findings indicate that anti-Nfasc155 IgG4 and anti-CNTN1 IgG4 act through completely different 
A

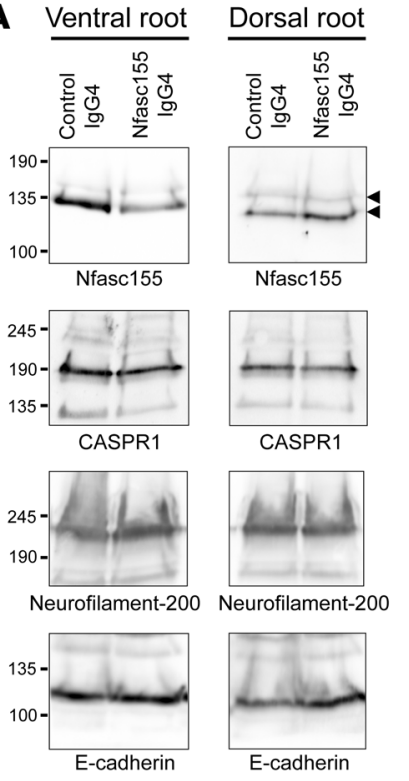

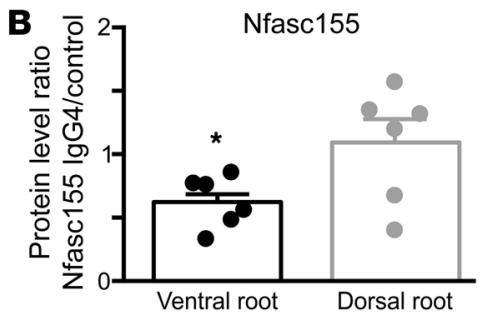
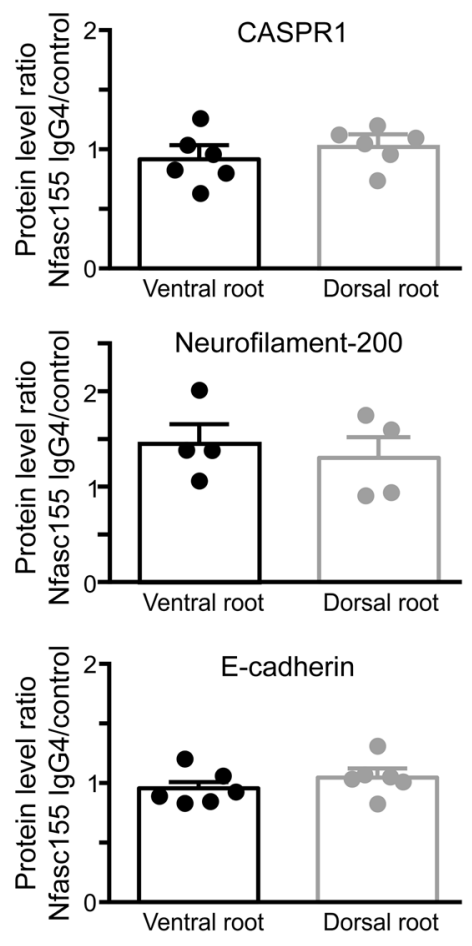

Figure 8. Autoantibodies induce the selective depletion of Nfasc155 in ventral spinal nerve. (A) Ventral and dorsal spinal nerve proteins $(250 \mu \mathrm{g})$ from adult animals injected with control lgG4 $(n=4-6)$ or anti-Nfasc155 IgC4 from patient CIDP1 $(n=4-6)$ for 3 weeks were separated on SDS-PAGE gels and immunoblotted with antibodies against Nfasc155, CASPR1, neurofilament-200, and E-cadherin. Arrowheads indicate Nfasc155-H and Nfasc155-L isoforms. (B) The levels of Nfasc155, CASPR1, neurofilament-200, and E-cadherin were evaluated in animals treated with anti-Nfasc155 IgG4 relatively to those in control animals in both ventral and dorsal spinal roots. Nfasc155 levels were significantly decreased in ventral spinal nerves, but not in dorsal spinal nerves $\left({ }^{*} P<0.05\right.$ by unpaired 2 -tailed Student's $t$ tests for 2 samples of equal variance). Molecular weight markers are shown on the left (in kilodaltons). Bars represent mean and SEM.

The passive transfer of anti-Nfasc155 IgG4 partially replicated the clinical deterioration seen in reactive patients. These latter present a neuropathy with a predominantly distal involvement associated with ataxia and disabling tremor $(4$, $8,36,37)$. The treated animals presented a severe phenotype akin to that of the patients but no tremor, and the electrophysiological and morphological findings suggested a predominant motor pathology. These apparent differences emphasized the limitations of animal models. Indeed, the determination of distal and proximal involvement, ataxia, or tremor relies on clinical tests difficult to perform in small mammals. Nonetheless, motor nerve conduction study revealed similitude between the patients and the animal model. Both showed conduction slowing and temporal dispersion on motor nerves $(9,24,25,36,40)$. The passive transfer of anti-Nfasc155 IgG4 thus replicated these electrophysiological features, and suggested that paranode alterations could be responsible for the conduction slowing observed in the patients. In keeping with this view, sural nerve biopsies from CIDP patients with antiNfasc155 IgG4 revealed specific alterations at paranodal regions with a loss of septate-like junctions (23-25), but did not show evidence of macrophage-mediated demyelination. The pathogenic mechanisms responsible for conduction slowing in patients with anti-Nfasc155 IgG4 thus appear different from those implicated in other CIDP patients, and further support the existence of nodo-paranodopathy (41). It is possible that paranodal demyelination, as observed in sural nerve biopsies $(24,25)$, may also participate in conduction slowing or loss in patients with anti-Nfasc155 IgG4. Here, we did not observe paranodal demyelination on semithin sections (data not shown), demonstrating that the paranodal junction dismantling itself is sufficient to generate conduction slowing. In fact, the genetic ablation of CASPR1 or CNTN1 abrogates paranodal adhesive junctions and results in important conduction slowing in the absence of myelin alterations (20,21), demonstrating that these subtle changes are sufficient to impact conduction velocity. The reason why we did not observe paranodal demyelination or sensory alterations in the animal model is unclear. One possibility is that a longer exposure time to autoantibody may be required to affect sensory axons or to induce paranodal detachment. Also these features may be more prominent in distal regions of the axon that could not be examined in our model.

The mechanisms of how anti-Nfasc155 IgG4 affects paranodal attachment appear to be distinct from those of anti-CNTN1 IgG4 . 
A

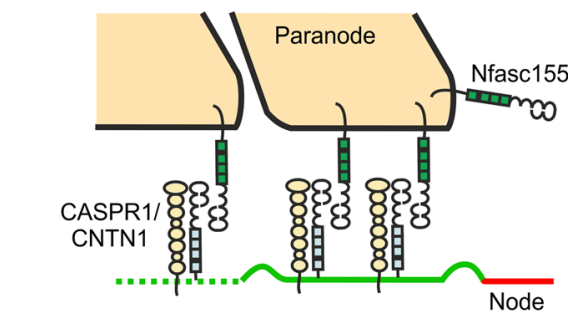

B

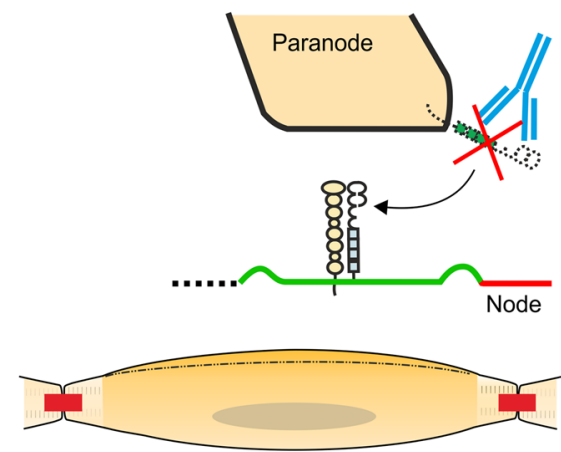

C

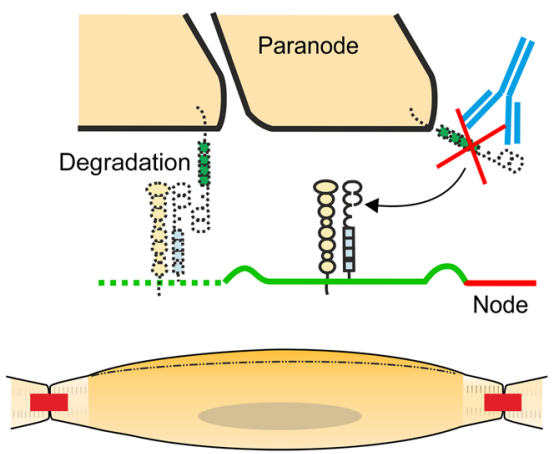

Figure 9. Schematic representation of the pathogenic mechanisms of anti-paranodal protein autoantibodies. (A) Representation of a mature node of Ranvier and myelinated axon. Myelin (yellow) covers the axon except at the node of Ranvier (red). At paranodal junctions (green), Nfasc155 interacts with its axonal partners CASPR1/CNTN1. Nfasc155 is also found at Schmidt-Lanterman incisures (dashed green lines). The Schwann cell nucleus is shown in gray. (B) In neonatal animals, the progressive enwrapping of axons by Schwann cells induces the formation of paranodal regions at node borders. The injection of anti-Nfasc155 lgG4 (blue) during the neonatal period does not affect myelination or node/paranode formation, but induces the depletion of Nfasc155, and thereby alters the formation of paranodal septate-like junctions. (C) At adult age, evidence suggests that the Nfasc155/CASPR1/CNTN1 complex is constantly renewed at paranodes possibly through degradation and replenishment mechanisms. The chronic infusion of anti-Nfasc155 IgG4 (blue) may preclude the regeneration of the paranodal axoglial junction by inducing Nfasc 155 depletion, and thereby alter paranode structure and conduction.

By contrast to anti-CNTN1 IgG4, anti-Nfasc155 IgG4 did not present a functional blocking activity and did not inhibit the interaction of rat Nfasc155 with the CNTN1/CASPR1 complex. Instead, anti-Nfasc155 IgG4 seemed to favor Nfasc155 aggregation and induce its depletion. A similar observation was observed with human isoforms, and anti-Nfasc155 IgG4 only modestly impaired human Nfasc155/CASPR1/CNTN1 interaction by comparison with anti-CNTN1 IgG4. Also, anti-Nfasc155 IgG4 did not dismantle the paranodal axoglial contact or penetrate paranodal regions like anti-CNTN1 IgG4 (29), but bound to the Schwann cell surface. This binding was seen with IgG4 from diverse anti-Nfasc155-positive patients and was abolished after immunoadsorption against Nfasc155, suggesting the presence of a cell surface Nfasc155 pool at the node vicinity and along the adherens junctions. The presence of Nfasc155 along adherens junctions in Schmidt-Lanterman incisures has already been reported several times $(34,42)$, but not its presence at the node vicinity. It is plausible that the high density of Nfasc155 at paranodes and incisures may preclude the detection of this surface protein pool. Indeed, human anti-Nfasc155 IgG4 predominantly stained paranodes and incisures after fixation and permeabilization, but staining at the node vicinity or adherens junctions was comparatively difficult to discern. Because the antibodies did not react against Nfasc186, it seems unlikely that this staining was due to binding to nodal Nfasc186. Also, IgG4 deposits did not colocalize with nodal axolemma markers, but surrounded the node. The significance of the surface Nfasc 155 pool is not yet clear. Our findings indicate that anti-Nfasc155 IgG4 binding at the node vicinity in neonatal animals is associated with a loss of paranode formation and that anti-Nfasc155 IgG4 induces Nfasc155 depletion. This suggests that antibody binding to the surface antigens favors its depletion and precludes CNTN1/CASPR1/ Nfasc 155 complex insertion into paranodes (Figure 9). In addition, our data suggest that anti-Nfasc155 IgG4 antibodies interfere with paranode stabilization in adults (Figure 9). Indeed, acute intraneural antibody injection had no effects once paranodes were formed in adult animals. By contrast, chronic antibody infusion induced clinical deficits, Nfasc155 depletion, and paranodal specialization loss, suggesting a blockade in axoglial junction maintenance. Evidence suggests that paranodal proteins are permanently renewed during the lifespan $(17,34,43)$. In genetic models, the ablation of the $N$ fasc gene induced a drastic decrease in Nfasc 155 protein level within 20 days (34) - a time frame that is in accord with the delay in observing important clinical and morphological abnormalities in our model, and with the aggressive onset observed in reactive patients. The timeline to reconstitute paranodal axoglial junctions is, however, yet unknown. Understanding the mechanisms regulating axoglial junction maintenance could be important to better delineate the factors regulating functional recovery.

Our in vitro assays clearly demonstrated that anti-Nfasc155 IgG4 does not have the potency to induce Nfasc155 internalization in HEK293 cells, Schwann cells, or oligodendrocytes. It thus seems unlikely that antibody-mediated endocytosis of Nfasc155 may be responsible for paranode alterations. Nevertheless, paranode alterations coincided with a significant and selective Nfasc155 depletion in both the neonatal and adult animal models. The mechanisms responsible for this protein loss are unclear. It seems unlikely that depletion of Nfasc155 could be a consequence of the loss of its consolidation at paranodes. Indeed, no decrease in Nfasc155 level was reported in animals with a truncated Nfasc155 form that lacks binding capacity to CNTN1/CASPR1 (44). One hypothesis is that anti-Nfasc155 IgG4 antibodies favor Nfasc155 proteolysis. A very recent study reported that Nfasc155 contains a thrombin cleavage site within its third fibronectin type III domain (45). Interestingly, this domain is located within the antigenic site recognized by anti-Nfasc155 IgG4. It could thus be possible that anti-Nfasc155 IgG4 favors Nfasc155 proteolytic cleavage either by affecting this domain structure or by favoring Nfasc155 aggregation. Another possibility could be that anti-Nfasc155 IgG4 dismantles the interaction of Nfasc 155 with other partners that are crucial for its stabilization at the membrane. These latter hypoth- 
eses remain to be demonstrated. Anyway, the loss of Nfasc155 was not combined with a depletion in CASPR1 despite the loss of CASPR1 accumulation at paranodes. In mice with a truncated Nfasc155 form, CASPR1 levels are also unchanged (44). This further confirms that CASPR1 clustering is strongly dependent on Nfasc155 $(34,46)$, but also indicates that expression and/or stability of CASPR1 is not dependent on its interaction with Nfasc155. During myelination, CASPR1 first appears in a diffuse manner along unmyelinated axons, then accumulates at the border of the myelinating Schwann cell in a spiral structure resembling a myelin wrap, and is finally consolidated at paranodes $(46,47)$. Here, the reverse phenomenon may occur, and Nfasc155 loss may induce the diffusion of CASPR1 along the axon.

In summary, this study demonstrates that Nfasc155 IgG4 antibodies impair the normal expression of $\mathrm{Nfasc} 155$ in the peripheral nerve, and provides strong evidence of their pathogenicity.

\section{Methods}

Patients' antibodies. Plasmapheresis fluids and sera were obtained from 3 CIDP patients with high titers of anti-Nfasc155 IgG4 antibodies and 1 CIDP patient with high titer of anti-CNTN1 IgG4 antibodies. All subjects gave written informed consent. Plasma from 2 healthy donors negative for CNTN1, Nfasc155, or CASPR1 antibodies was used as control (Etablissement Français du Sang). IgG4 antibodies were purified using CaptureSelect affinity matrix (Thermo Fisher Scientific) as previously described (29). Fractions were concentrated using a Pierce protein concentrator (Thermo Fisher Scientific), then dialyzed to artificial CSF: $126 \mathrm{mM} \mathrm{NaCl}, 3 \mathrm{mM} \mathrm{KCl}, 2 \mathrm{mM} \mathrm{CaCl}, 2 \mathrm{mM} \mathrm{MgSO}_{4}$, $1.25 \mathrm{mM} \mathrm{NaH}_{2} \mathrm{PO}_{4}, 26 \mathrm{mM} \mathrm{NaHCO}_{3}$, and $10 \mathrm{mM}$ dextrose, $\mathrm{pH}$ 7.4-7.5. Fractions were finally diluted to a concentration of $10 \mathrm{mg} / \mathrm{ml}$ in artificial CSF and sterilized by filtration. Fraction purity was verified by Western blot and cell-binding assay, but also by ELISA and on rat sciatic nerve as previously described (29) (Supplemental Figures 1 and 2).

Immunoadsorption. IgG Fc-conjugated human Nfasc155 was purified as previously described (48). Five hundred microliters of packed agarose protein A beads (Sigma-Aldrich) were incubated overnight at $4^{\circ} \mathrm{C}$ with a saturating quantity of Fc-Nfasc155 $(1 \mathrm{~g})$, and then the beads were extensively washed with PBS. One hundred micrograms of purified anti-Nfasc155 IgG4 was then incubated overnight at $4^{\circ} \mathrm{C}$ with $100 \mu \mathrm{l}$ of $\mathrm{Nfasc} 155$ beads for each patient. Beads were sedimented by centrifugation, the supernatants were collected, and protein concentrations were quantified. This procedure resulted in the depletion of $80 \%, 62 \%$, and $42 \%$ of the proteins in the CIDP1, CIDP2, and CIDP3 samples, respectively. These samples were used for Western blot, ELISA, immunohistology on sciatic nerve and HEK293 cells, and nerve incubation assays.

Western blot. Purified antibody samples were denatured in SDS sample buffer for 2 minutes at $92^{\circ} \mathrm{C}$, loaded on $7.5 \%$ SDS-PAGE gels, transferred, and immunoblotted with mouse monoclonal antibodies against human IgG1 (1:1000; 4E3, Abcam), IgG2 (1:1000; HP6014, Abcam), IgG3 (1:1000; HP-6050, Sigma-Aldrich), or IgG4 (1:5000; HP6035, Abcam). Brain and cerebellum protein samples were prepared as previously described (4). Sciatic nerves from neonatal or adult rats were directly solubilized in SDS sample buffer and complete protease inhibitor cocktail (Roche), then heated for 2 minutes at $90^{\circ} \mathrm{C}$. The samples were centrifuged for 10 minutes at $750 \mathrm{~g}$. Protein concentration was determined using a BCA kit (Bio-Rad). Samples
$(100 \mu \mathrm{g})$ were loaded on a 5\% SDS-PAGE gel, transferred onto nitrocellulose membranes, and immunoblotted with mouse antibodies against E-cadherin (1:2000; 610181, BD Biosciences) or rabbit antisera against pan-neurofascin (1:1000; Ab184378, Abcam), CASPR1 (1:10,000; gift of Laurence Goutebroze, INSERM UMR-S839, Paris, France) (49), Nfasc155 (1:1000; 15035, Cell Signaling Technology), or neurofilament-200 (1:10,000; Ab1987, Abcam) overnight at $4^{\circ} \mathrm{C}$. Immunoreactivity was revealed with the appropriate peroxidasecoupled secondary antibodies (1:5000; Jackson ImmunoResearch Laboratories) for 1 hour, washed several times and revealed using a BM chemiluminescence kit (Sigma-Aldrich).

Cell aggregation assay. HEK293 cells were grown in DMEM containing 10\% FCS (Thermo Fisher Scientific) and were transiently transfected using JetPEI (Polyplus-transfection) with mCherryconjugated rat Nfasc155 or with both rat CNTN1 and GFP-conjugated rat CASPR1. For negative control, cells were solely transfected with peGFP-N1 (Clontech). One day after transfection, cells were washed with PBS, and trypsinated with $0.25 \%$ trypsin in PBS for 5 minutes at $37^{\circ} \mathrm{C}$. Cells were centrifuged and suspended in Opti-MEM (Thermo Fisher Scientific). Cells were then mixed together in a 1:1 ratio $(400,000$ cells $/ \mathrm{ml})$ in the presence of $10 \mu \mathrm{g}$ of purified antibodies (final concentration $20 \mu \mathrm{g} / \mathrm{ml}$ ), and were agitated at $100 \mathrm{rpm}$ for 2 hours at $37^{\circ} \mathrm{C}$. Fifty microliters of cell suspension was then mounted between slides and coverslip, and was immediately observed using an ApoTome fluorescence microscope at the $\times 10$ objective. Aggregates were defined as clusters of at least 4 cells. Aggregates showing interactions between green and red cells were considered as cell clusters with contacts. In addition, the percentage of green cells was quantified in each cell cluster. Four experiments were performed for each condition, and a minimum of 40 cell clusters were quantified per experiment.

Animals and surgery. For in vitro nerve incubation experiments, 6-week-old male Lewis rats (purchased from Janvier Laboratories) were euthanized, and the sciatic nerves were quickly dissected out, desheathed, cut in 1-cm segments, and incubated for 1 hour or 3 hours with $10 \mu \mathrm{g}$ of purified anti-Nfasc155 IgG4 diluted in artificial CSF (final concentration $50 \mu \mathrm{g} / \mathrm{ml}$ ). Nerve segments were washed $5 \mathrm{~min}$ utes with artificial CSF, pH 7.4 or pH 2.0, then washed 5 minutes twice with normal artificial CSF, fixed in $2 \%$ paraformaldehyde in PBS for 1 hour at $4^{\circ} \mathrm{C}$, and processed for immunolabeling.

For injections in neonatal animals, newborn pups received a single i.p. injection of $250 \mu \mathrm{g}$ of control IgG4 or anti-Nfasc155 IgG4 in artificial CSF $(25 \mu \mathrm{l})$. Animals were then sacrificed at 2, 4, or 6 days after the injection. In some experiments, newborn pups received daily i.p. injection of $250 \mu \mathrm{g}$ of control IgG4 or anti-Nfasc155 IgG4 in artificial CSF $(25 \mu \mathrm{l})$ from PO to P6, and were sacrificed at P4 or P6.

For intraneural injections, anesthesia was induced and maintained with Vetflurane (Virbac). Animals also received a subcutaneous injection of buprenorphine for pain relief. The right sciatic nerve was exposed at the level of the sciatic notch and injected with $2 \mu \mathrm{l}$ of antibody $(10 \mu \mathrm{g} / \mu \mathrm{l})$ using a glass micropipette. One or three days after surgery, injected nerves were processed for immunolabeling as detailed below.

For intrathecal infusion, male Lewis rats (10 weeks old, $300 \mathrm{~g}$ ) were anesthetized with Vetflurane and received a subcutaneous injection of buprenorphine for pain relief. Intrathecal polyurethane catheters (Instech, BTPU-027; 0.43 inner diameter, $0.69 \mathrm{~mm}$ outer diameter, $8.0 \mathrm{~cm}$ long) were connected to a vascular access button 
(Instech, VAB62BS/25). The catheters were inserted through a puncture of the atlanto-occipital membrane toward the lumbar region as previously described (50), and the vascular button was fixed under the skin on the head of the animal. After recovery for 1 week, the animals received daily intrathecal injections of $10 \mu \mathrm{l}$ of purified control IgG4 or anti-Nfasc155 IgG4 $(10 \mathrm{mg} / \mathrm{ml})$. Injections were performed daily for 5 consecutive days followed by a 2-day break for 3 weeks. Animals were weighed and examined daily for clinical signs. Clinical signs were graded as follows: 0 , no illness; 1 , tail tip hanging; 2 , limp tail; 3 , tail paralysis; 4 , gait ataxia; 5 , mild paraparesis; 6 , severe paraparesis; 7, paraplegia; 8 , tetraparesis; 9 , moribund; 10 , death. To monitor gait abnormalities, animals were allowed to walk freely along a 2-meterlong surface (Supplemental Video 1). For footprint analysis, the animal hind paws were dipped in ink and the animals walked down a corridor on white paper. The angles of the footprints were measured with ImageJ version $1.43 \mathrm{u}$ software (NIH). The hind-limb grip strength was monitored before antibody injection and at 18 days using a BIO-GS3 grip test (Bioseb). For histopathology and electrophysiology, animals were sacrificed after 21 days of injections.

Immunolabeling and histopathology. Sciatic nerves and L6 ventral and dorsal spinal nerves were dissected out and fixed in $2 \%$ paraformaldehyde in PBS for 1 hour at $4^{\circ} \mathrm{C}$, then rinsed in PBS. Axons were gently teased, dried on glass slides, and stored at $-20^{\circ} \mathrm{C}$. Teased fibers were permeabilized by immersion in $-20^{\circ} \mathrm{C}$ acetone for 10 minutes, blocked at room temperature for 1 hour with PBS containing $5 \%$ fish skin gelatin and $0.1 \%$ Triton $\mathrm{X}-100$, then incubated overnight at $4{ }^{\circ} \mathrm{C}$ with primary antibodies: rabbit antisera against Nfasc186 (1:500; gift of Alex Gow, Wayne State University, Detroit, Michigan, USA) (51), ankyrin-G (1:500; gift of Gisèle Alcaraz, GMGF, INSERM, UMR-S910, Marseille, France) (52), or CASPR1 (1:2000); mouse monoclonal antibodies against $\mathrm{Na}_{\mathrm{v}}$ channels (1:500; K58/35, Sigma-Aldrich) or $\beta$-catenin (MAB2081, Merck Millipore); and goat antibody against CNTN1 (1:2000; R\&D Systems). The slides were then washed several times and incubated with Alexa-conjugated donkey antisera against rabbit, mouse, human, or goat IgG (1:500; Jackson ImmunoResearch Laboratories) or FITC-conjugated mouse monoclonal antibodies against human IgG1 (1:500; clone 4E3, ab99772, Abcam), IgG2 (1:500; clone HP6014, ab99788, Abcam), IgG3 (1:500; clone HP6050, F4641, SigmaAldrich), or IgG4 (1:500; clone HP6025, ab99821, Abcam). Slides were mounted with Mowiol plus 2\% DABCO, and examined using an ApoTome fluorescence microscope (Carl Zeiss MicroImaging GmbH). Digital images were manipulated into figures with CorelDraw and Corel Photo-Paint. Teased fibers were analyzed from 11 animals for each group. For quantification of paranodal alterations, the percentage of nodes showing loss of paranodal regions was quantified in 11 animals ( 100 axons counted in total).

For histopathological analysis, nerves were fixed in $2.5 \%$ glutaraldehyde in $0.1 \mathrm{M}$ sodium cacodylate buffer plus $0.16 \mathrm{M}$ sucrose overnight at $4^{\circ} \mathrm{C}$ and postfixed in $1 \% \mathrm{OsO}_{4}$ in $0.1 \mathrm{M}$ sodium cacodylate buffer for 1 hour. Nerves were dehydrated and embedded in epoxy resin. Transverse semithin sections were stained with toluidine blue and examined by light microscopy. Longitudinal ultrathin sections were examined by electron microscopy as previously described (25).

Oligodendrocyte culture. Oligodendrocyte precursor cells were prepared from rats as previously described (4). Oligodendrocyte precursor cells were plated into 12 -well plates containing poly-L-lysine-coated 12 -mm coverslips at a density of 250,000 cells per well. To allow differentiation into oligodendrocytes, cells were maintained in 50\% neurobasal medium (Thermo Fisher Scientific), 50\% DMEM, $4 \mathrm{mM}$ L-glutamine, $1 \mathrm{mM}$ sodium pyruvate, penicillin/streptomycin, $2 \times$ B27 supplement, $1 \times \mathrm{N}_{2}$ supplement, $100 \mu \mathrm{g} / \mathrm{ml} \mathrm{BSA}, 40 \mathrm{ng} / \mathrm{ml}$ triiodothyronine, $5 \mu \mathrm{g} / \mathrm{ml} \mathrm{N}$-acetylcysteine, and $10 \mathrm{ng} / \mathrm{ml}$ biotin. After 10 days in vitro, oligodendrocytes were incubated with CIDP sera (1:200) in neurobasal medium for 1 hour at $37^{\circ} \mathrm{C}$, washed 2 minutes with neurobasal $\mathrm{pH} 7.4$ or $\mathrm{pH}$ 2.0, then washed twice 5 minutes with normal neurobasal, and fixed with $2 \%$ paraformaldehyde for 20 minutes. Cells were permeabilized with blocking solution for 20 minutes, incubated for 1 hour at room temperature with rat antibodies against myelin basic protein (1:100; MCA409S, Bio-Rad), and revealed with secondary antibodies.

Electrophysiology. Recordings were performed at 21 days as previously described (48). Briefly, the L6 spinal nerves and sciatic nerves were dissected out and transferred into artificial CSF. CAP recordings were made at $35^{\circ} \mathrm{C}$ in a 3-compartment recording chamber. Conduction velocities were estimated from latencies and were calculated at maximal and at half maximal CAP amplitude.

Statistics. Statistical significance was assessed by unpaired 2-tailed Student's $t$ tests, Kolmogorov-Smirnov tests, or 1-way ANOVA followed by Bonferroni's post hoc test using GraphPad Prism (GraphPad Software). $P$ values less than 0.05 were considered significant.

Study approval. All animal experiments were in line with the European Community's guiding principles on the care and use of animals (2010/63/EU) and were approved by the local ethical committee (Comité d'Ethique pour l'Expérimentation Animale Languedoc Roussillon no. 36, Biocampus, Montpellier, France) and by the "ministére de l'éducation nationale de l'enseignement supérieur et de la recherche" (APAFIS 3847-2016012610089856v8).

\section{Author contributions}

CM, LQ, CL, MP, MM, JMV, II, and JJD drafted and revised the manuscript. JJD, LQ, and II conceived and designed the study. CM, LQ, CL, MP, MM, JMV, II, and JJD acquired data. CM, LQ, CL, MP, MM, JMV, II, and JJD analyzed and interpreted data.

\section{Acknowledgments}

We thank Laurence Goutebroze, Alex Gow, and Giséle Alcaraz for gifts of antibodies, and Axel Fernandez for technical assistance. JJD, LQ, and II acknowledge grant support from the Agence Nationale pour la Recherche; the Centro para la Investigación Biomédica en Red (CIBERER; ACAMIN project) under the frame of E-Rare-2; the ERA-Net for Research on Rare Diseases; the Association Française contre les Myopathies (grant 21532 to JJD); the GBS-CIDP Foundation International (to CL); CSL Behring's grant in immunology (to JJD); the Pla Estratègic de Recerca i Innovació en Salut (PERIS) 2016-2020 of the Departament de Salut of the Generalitat de Catalunya (SLT006/17/00131 to LQ); and the Fondo de Investigaciones Sanitarias, Instituto de Salud Carlos III, Ministry of Health, Spain (PI16/000627, FEDER funds, to LQ; and FIS 13/00937 to II).

Address correspondence to: Jérôme J. Devaux, Institute for Neurosciences of Montpellier, INSERM U1051, Montpellier University, Hopital Gui de Chauliac, 80 avenue Augustin Fliche, 34295 Montpellier Cedex 5, France. Phone: 33.4.99.63.60.95; Email: jerome. devaux@inserm.fr. 
1. Dalakas MC, Medscape. Advances in the diagnosis, pathogenesis and treatment of CIDP. Nat Rev Neurol. 2011;7(9):507-517.

2. Querol L, et al. Antibodies against peripheral nerve antigens in chronic inflammatory demyelinating polyradiculoneuropathy. Sci Rep. 2017;7(1):14411

3. Delmont E, et al. Autoantibodies to nodal isoforms of neurofascin in chronic inflammatory demyelinating polyneuropathy. Brain. 2017;140(7):1851-1858.

4. Devaux JJ, et al. Neurofascin-155 IgG4 in chronic inflammatory demyelinating polyneuropathy. Neurology. 2016;86(9):800-807.

5. Doppler K, et al. Auto-antibodies to contactinassociated protein 1 (Caspr) in two patients with painful inflammatory neuropathy. Brain 2016;139 (pt 10):2617-2630.

6. Doppler K, et al. Destruction of paranodal architecture in inflammatory neuropathy with anti-contactin-1 autoantibodies. J Neurol Neurosurg Psychiatry. 2015;86(7):720-728.

7. Miura Y, et al. Contactin 1 IgG4 associates to chronic inflammatory demyelinating polyneuropathy with sensory ataxia. Brain.

2015;138(pt 6):1484-1491

8. Ng JK, et al. Neurofascin as a target for autoantibodies in peripheral neuropathies. Neurology. 2012;79(23):2241-2248.

9. Querol L, et al. Neurofascin IgG4 antibodies in CIDP associate with disabling tremor and poor response to IVIg. Neurology. 2014;82(10):879-886.

10. Querol L, et al. Antibodies to contactin-1 in chronic inflammatory demyelinating polyneuropathy. Ann Neurol. 2013;73(3):370-380.

11. Painous C, López-Pérez MÁ, Illa I, Querol L. Head and voice tremor improving with immunotherapy in an anti-NF155 positive CIDP patient. Ann Clin Transl Neurol. 2018;5(4):499-501.

12. Querol L, et al. Rituximab in treatment-resistant CIDP with antibodies against paranodal proteins. Neurol Neuroimmunol Neuroinflamm. 2015;2(5):e149.

13. Faivre-Sarrailh C, Devaux JJ. Neuro-glial interactions at the nodes of Ranvier: implication in health and diseases. Front Cell Neurosci. 2013;7:196.

14. Freeman SA, Desmazières A, Fricker D, Lubetzki C, Sol-Foulon N. Mechanisms of sodium channe clustering and its influence on axonal impulse conduction. Cell Mol Life Sci. 2016;73(4):723-735.

15. Feinberg K, et al. A glial signal consisting of gliomedin and $\mathrm{NrCAM}$ clusters axonal $\mathrm{Na}^{+}$channels during the formation of nodes of Ranvier. Neuron. 2010;65(4):490-502.

16. Amor V, et al. Long-term maintenance of $\mathrm{Na}^{+}$ channels at nodes of Ranvier depends on glial contact mediated by gliomedin and NrCAM. J Neurosci. 2014;34(15):5089-5098.

17. Taylor AM, Saifetiarova J, Bhat MA. Postnatal loss of neuronal and glial neurofascins differentially affects node of Ranvier maintenance and myelinated axon function. Front Cell Neurosci. 2017;11:11

18. Thaxton C, Pillai AM, Pribisko AL, Dupree JL, Bhat MA. Nodes of Ranvier act as barriers to restrict invasion of flanking paranodal domains in myelinated axons. Neuron. 2011;69(2):244-257.
19. Vallat JM, et al. Subacute nodopathy with conduction blocks and anti-neurofascin 140/186 antibodies: an ultrastructural study. Brain. 2018;141(7):e56.

20. Bhat MA, et al. Axon-glia interactions and the domain organization of myelinated axons requires neurexin IV/Caspr/Paranodin. Neuron. 2001;30(2):369-383.

21. Boyle ME, Berglund EO, Murai KK, Weber L, Peles E, Ranscht B. Contactin orchestrates assembly of the septate-like junctions at the paranode in myelinated peripheral nerve. $\mathrm{Neu}$ ron. 2001;30(2):385-397.

22. Sherman DL, et al. Neurofascins are required to establish axonal domains for saltatory conduction. Neuron. 2005;48(5):737-742.

23. Koike $\mathrm{H}$, et al. Paranodal dissection in chronic inflammatory demyelinating polyneuropathy with anti-neurofascin-155 and anti-contactin-1 antibodies. J Neurol Neurosurg Psychiatry. 2017;88(6):465-473.

24. Kuwahara M, et al. Electron microscopic abnormality and therapeutic efficacy in chronic inflammatory demyelinating polyneuropathy with anti-neurofascin 155 immunoglobulin G4 antibody. Muscle Nerve. 2018;57(3):498-502

25. Vallat JM, et al. Paranodal lesions in chronic inflammatory demyelinating polyneuropathy associated with anti-Neurofascin 155 antibodies. Neuromuscul Disord. 2017;27(3):290-293.

26. Huijbers MG, et al. The expanding field of IgG4-mediated neurological autoimmune disorders. Eur J Neurol. 2015;22(8):1151-1161.

27. Querol L, Devaux J, Rojas-Garcia R, Illa I. Autoantibodies in chronic inflammatory neuropathies: diagnostic and therapeutic implications. Nat Rev Neurol. 2017;13(9):533-547.

28. Labasque M, Hivert B, Nogales-Gadea G, Querol L, Illa I, Faivre-Sarrailh C. Specific contactin $\mathrm{N}$-glycans are implicated in neurofascin binding and autoimmune targeting in peripheral neuropathies. J Biol Chem. 2014;289(11):7907-7918.

29. Manso C, Querol L, Mekaouche M, Illa I, Devaux JJ. Contactin-1 IgG4 antibodies cause paranode dismantling and conduction defects. Brain. 2016;139(pt 6):1700-1712.

30. Eshed Y, et al. Gliomedin mediates Schwann cell-axon interaction and the molecular assembly of the nodes of Ranvier. Neuron. 2005;47(2):215-229.

31. Schafer DP, Custer AW, Shrager P, Rasband MN Early events in node of Ranvier formation during myelination and remyelination in the PNS. Neuron Glia Biol. 2006;2(2):69-79.

32. Smith CE, Atchabahian A, Mackinnon SE, Hunter DA. Development of the blood-nerve barrier in neonatal rats. Microsurgery. 2001;21(7):290-297.

33. Pomicter AD, et al. Novel forms of neurofascin 155 in the central nervous system: alterations in paranodal disruption models and multiple sclerosis. Brain. 2010;133(pt 2):389-405

34. Pillai AM, Thaxton C, Pribisko AL, Cheng JG, Dupree JL, Bhat MA. Spatiotemporal ablation of myelinating glia-specific neurofascin (Nfasc NF155) in mice reveals gradual loss of paranodal axoglial junctions and concomitant disorganization of axonal domains. J Neurosci Res. 2009;87(8):1773-1793.
35. Franques J, Chapon F, Devaux J, Mathis S. Teaching NeuroImages: cranial nerve hypertrophy in IgG4 anti-neurofascin 155 antibody-positive polyneuropathy. Neurology. 2017;88(7):e52.

36. Ogata $\mathrm{H}$, et al. Characterization of IgG4 antineurofascin 155 antibody-positive polyneuropathy. Ann Clin Transl Neurol. 2015;2(10):960-971.

37. Kadoya M, et al. IgG4 anti-neurofascin155 antibodies in chronic inflammatory demyelinating polyradiculoneuropathy: clinical significance and diagnostic utility of a conventional assay. J Neuroimmunol. 2016;301:16-22.

38. Martinez-Martinez L, et al. Anti-NF155 chronic inflammatory demyelinating polyradiculoneuropathy strongly associates to HLA-DRB15. J Neuroinflammation. 2017;14(1):224.

39. Yan W, et al. Antibodies to neurofascin exacerbate adoptive transfer experimental autoimmune neuritis. J Neuroimmunol. 2014;277(1-2):13-17.

40. Koike H, et al. Restoration of a conduction block after the long-term treatment of CIDP with anti-neurofascin 155 antibodies: follow-up of a case over 23 years. Intern Med 2018;57(14):2061-2066.

41. Uncini A, Vallat JM. Autoimmune nodo-paranodopathies of peripheral nerve: the concept is gaining ground. J Neurol Neurosurg Psychiatry. 2018;89(6):627-635.

42. Tait S, et al. An oligodendrocyte cell adhesion molecule at the site of assembly of the paranodal axo-glial junction. J Cell Biol. 2000;150(3):657-666.

43. Desmazieres A, Zonta B, Zhang A, Wu LM, Sherman DL, Brophy PJ. Differential stability of PNS and CNS nodal complexes when neuronal neurofascin is lost. JNeurosci. 2014;34(15):5083-5088.

44. Thaxton $\mathrm{C}$, et al. In vivo deletion of immunoglobulin domains 5 and 6 in neurofascin (Nfasc) reveals domain-specific requirements in myelinated axons. JNeurosci. 2010;30(14):4868-4876.

45. Dutta DJ, et al. Regulation of myelin structure and conduction velocity by perinodal astrocytes. Proc Natl Acad Sci U S A. 2018;115(46):11832-11837.

46. Eisenbach M, et al. Differential clustering of Caspr by oligodendrocytes and Schwann cells. J Neurosci Res. 2009;87(15):3492-3501.

47. Pedraza L, Huang JK, Colman DR. Organizing principles of the axoglial apparatus. Neuron. 2001;30(2):335-344

48. Lonigro A, Devaux JJ. Disruption of neurofascin and gliomedin at nodes of Ranvier precedes demyelination in experimental allergic neuritis. Brain. 2009;132(pt 1):260-273.

49. Menegoz M, et al. Paranodin, a glycoprotein of neuronal paranodal membranes. Neuron. 1997;19(2):319-331.

50. Yaksh TL, Rudy TA. Chronic catheterization of the spinal subarachnoid space. Physiol Behav. 1976;17(6):1031-1036.

51. Southwood C, He C, Garbern J, Kamholz J, Arroyo E, Gow A. CNS myelin paranodes require Nkx6-2 homeoprotein transcriptional activity for normal structure. J Neurosci. 2004;24(50):11215-11225.

52. Bouzidi M, et al. Interaction of the Nav1.2a subunit of the voltage-dependent sodium channel with nodal ankyrinG. In vitro mapping of the interacting domains and association in synaptosomes. J Biol Chem. 2002;277(32):28996-29004. 